\title{
On the analysis of a coupled kinetic-fluid model with local alignment forces
}

\author{
José A. Carrillo ${ }^{\mathrm{a}, *}$, Young-Pil Choi ${ }^{\text {a }}$, Trygve K. Karper ${ }^{\mathrm{b}}$ \\ ${ }^{a}$ Department of Mathematics, Imperial College London, London SW7 2AZ, United Kingdom \\ ${ }^{\mathrm{b}}$ Department of Mathematical Sciences, Norwegian University of Science and Technology, Trondheim, N-7491, Norway
}

Received 21 November 2013; received in revised form 6 October 2014; accepted 16 October 2014

Available online 22 October 2014

\begin{abstract}
This paper studies global existence, hydrodynamic limit, and large-time behavior of weak solutions to a kinetic flocking model coupled to the incompressible Navier-Stokes equations. The model describes the motion of particles immersed in a Navier-Stokes fluid interacting through local alignment. We first prove the existence of weak solutions using energy and $L^{p}$ estimates together with the velocity averaging lemma. We also rigorously establish a hydrodynamic limit corresponding to strong noise and local alignment. In this limit, the dynamics can be totally described by a coupled compressible Euler - incompressible Navier-Stokes system. The proof is via relative entropy techniques. Finally, we show a conditional result on the large-time behavior of classical solutions. Specifically, if the mass-density satisfies a uniform in time integrability estimate, then particles align with the fluid velocity exponentially fast without any further assumption on the viscosity of the fluid.
\end{abstract}

(C) 2015 L'Association Publications de l'Institut Henri Poincaré. Published by Elsevier B.V. All rights reserved.

Keywords: Kinetic-fluid coupled equations; Asymptotic behavior; Flocking; Hydrodynamical limit

\section{Introduction}

In the animal kingdom, one can find several species where the action of individuals leads to large coherent structures and where there are no external forces or "leader" guiding the interaction. Perhaps the most famous examples are flocks of birds, schools of fish, or insect swarms. However, similar phenomena in self-organization are also relevant for bacteria, in robotic engineering, and in material science. The past decade has witnessed a massive growth in the attempts to develop mathematical models capturing these types of phenomena. These models are usually based on incorporating different mechanisms of interaction between the individuals such as local repulsion, long-range attraction, and alignment. These Individual Based Models lead to macroscopic descriptions by means of mean-field limit scalings, see [7] for a review. These continuum descriptions can be written as kinetic equations in which there is a mechanism of interaction in the velocity or orientation vector. A very simple idea implementing the consensus

\footnotetext{
* Corresponding author.

E-mail addresses: carrillo@ imperial.ac.uk (J.A. Carrillo), young-pil.choi@imperial.ac.uk (Y.-P. Choi), karper@gmail.com (T.K. Karper).
} 
mechanism in velocity was introduced by Cucker and Smale in [9] and improved recently in [28]. These models take into account nonlocal interactions of the particles by averaging in velocity space. Here, we will focus on a much stronger local averaging of the velocity vector and the effect of a fluid in the tendency to consensus. We will explain the relation to these classical models of alignment below.

The model under consideration governs the motion of particles immersed in a Navier-Stokes fluid interacting through local alignment. By local alignment, we mean that each particle actively tries to align its velocity to that of its closest neighbors. The particles and fluid are coupled through linear friction. If we let $f=f(x, \xi, t)$ be the one-particle distribution function at a spatial periodic domain $x \in \mathbb{T}^{3}, \xi \in \mathbb{R}^{3}$ at time $t$, and $u=u(x, t)$ be the bulk velocity of fluid, then our model reads

$$
\begin{aligned}
& \partial_{t} f+\xi \cdot \nabla_{x} f=\alpha \nabla_{\xi} \cdot[(\xi-u) f]+\beta \nabla_{\xi} \cdot\left[\left(\xi-u_{f}\right) f\right]+\sigma \Delta_{\xi} f \\
& \partial_{t} u+u \cdot \nabla_{x} u+\nabla_{x} p=\mu \Delta_{x} u-\alpha \rho_{f}\left(u-u_{f}\right) \\
& \nabla_{x} \cdot u=0
\end{aligned}
$$

subject to initial data

$$
f(x, \xi, 0)=f_{0}(x, \xi), \quad u(x, 0)=u_{0}(x),
$$

where $\alpha, \beta, \sigma>0$ are constants, and $\rho_{f}$ and $u_{f}$ denote the average local density and velocity, respectively

$$
\rho_{f}:=\int_{\mathbb{R}^{3}} f d \xi, \quad \rho_{f} u_{f}:=\int_{\mathbb{R}^{3}} \xi f d \xi .
$$

The model (1.1) contains as particular cases two previously studied models in the literature. If $\beta=0$, the model reduces to the fluid-particle model studied in $[16,17]$, see also $[3,8,18,26,27]$. They analyzed the existence of weak solutions and their hydrodynamic limit. On the other hand, if $\alpha=0,(1.1)$ decouples and becomes the kinetic flocking model studied in [21-23]. This latter series of papers establishes existence of weak solutions and hydrodynamic limit, but leaves out the question of large-time behavior.

In this paper, we shall be concerned with the case $\alpha, \beta>0$. This introduces new difficulties compared to the previous studies, requiring non trivial arguments to overcome them. To prove existence of weak solutions to (1.1), the main challenges are posed by the product $f u_{f}$ and the lack of regularity on $u$. In the first case, weak compactness of $f u_{f}$ is not trivial as there does not seem to be any available regularity in a spatial domain. Moreover, $u_{f}$ is only defined on regions with $\varrho_{f}>0$ and hence does not belong to any $L^{p}$-space. In this paper, we will obtain the needed compactness from the velocity averaging lemma together with some technical arguments. This part of the proof will be similar to the existence proof in [21] for (1.1) with $\alpha=0$. However, the coupling with the Navier-Stokes equations introduces new problems that are not straightforward to handle.

Since Eq. (1.1) is posed in $2 d+1$ dimensions, finding an approximate solution is computationally expensive. For this reason, it is of interest to identify regimes where the complexity of the equations reduces. In this paper, we shall rigorously identify one such regime corresponding to strong noise and local alignment. That is, the case where $\beta \sim \sigma \sim \varepsilon^{-1}$, where $\varepsilon$ is a small number. We will establish that in this case $f$ is close to a thermodynamical equilibrium $f \sim c_{0} \varrho_{f} e^{-\left|u_{f}-\xi\right|^{2} / 2}$ and that the dynamics can be well approximated by a compressible Euler equation for $\left(\varrho_{f}, u_{f}\right)$ coupled to the incompressible Navier-Stokes equations for $u$ (see Section 2.2 for clarity). We will achieve this result by establishing a relative entropy inequality. Though this type of inequality was originally devised in [10] to prove weak-strong uniqueness results, it has also been successfully applied to hydrodynamic limits for kinetic equations [14,24,29]. The perhaps most relevant study is [22], where (1.1) with $\alpha=0$ is studied. However, with $\beta>0$, deriving a relative entropy bound is more involved and requires completely new arguments that does not have a kin in the literature.

For the estimates of large-time behavior of solutions, when $\beta=0$, i.e., no local alignment force, the particle-fluid equations (1.1) reduce to the Vlasov-Navier-Stokes-Fokker-Planck equations. For this system, classical solutions near Maxwellians converging asymptotically to them were constructed in [15]. More recently, the incompressible Euler-Fokker-Planck equations $(\beta=0$ and $\mu=0)$ were treated in [5] showing the existence of a unique classical solution near Maxwellians converging to them. On the other hand, without the diffusive term $(\sigma=0)$, the particle-fluid system has no trivial equilibria, and as a consequence the previous arguments used in $[5,15]$ for the estimates of largetime behavior can not be applied. The large-time behavior of the Vlasov-Navier-Stokes equations, to our knowledge, 
has only been studied in periodic spatial domain [1,2]. By replacing the Cucker-Smale alignment force in [1] by the local alignment one, we will show the emergence of alignment between fluid and particles as time evolves. Our strategy to analyse the large-time behavior can be applied to the system discussed in [1], improving their results by weakening the assumptions. Unfortunately, our framework can not be applied to the Vlasov-Navier-Stokes equations in the whole space, since we do not know how to get information from the dissipation of the fluid equation replacing the Sobolev inequality.

Let us now give some explanation for the term local alignment and how this pertains to the Cucker-Smale flocking model. In the previous decade, Cucker \& Smale [9] introduced a Newtonian-type flocking model using $\ell^{2}$-based arguments:

$$
\frac{d x_{i}}{d t}=\xi_{i}, \quad \frac{d \xi_{i}}{d t}=\sum_{j=1}^{N} \psi_{i j}^{c s}\left(\xi_{j}-\xi_{i}\right), \quad t>0, i \in\{1, \ldots, N\},
$$

where $x_{i}(t) \in \mathbb{R}^{d}$ and $\xi_{i}(t) \in \mathbb{R}^{d}$ are the position and velocity of $i$-th particles at time $t$, respectively and were $\psi_{i j}^{c s}$ is a communication weight between particles defined by

$$
\psi_{i j}^{c s}:=\frac{1}{N} \psi^{c s}\left(\left|x_{i}-x_{j}\right|\right), \quad i, j \in\{1, \ldots, N\} .
$$

Subsequently, this flocking model and its invariants have been extensively studied in a vast number of papers such as $[4,6,19,20]$ to mention a few. However, more recently Motsch and Tadmor pointed out several deficiencies with the Cucker-Smale model, and suggested a new model which take into account not only distance between particles but also their relative distance [28]. More precisely, they considered a nonsymmetric communication weight normalized with a local average density:

$$
\psi_{i j}^{m t}:=\frac{\psi_{i j}^{c s}}{\sum_{k=1}^{N} \psi_{i k}^{c s}} .
$$

As a result, the Motsch-Tadmor model does not involve any explicit dependence on the number of particles. Since $\psi_{i j}^{m t}$ is nonsymmetric, they introduce a new tools based on the notion of active sets to estimate the flocking behavior of particles.

On the other hand, when the number of particles goes to infinity, $N \rightarrow \infty$, one can formally derive a mesoscopic description for system (1.4)-(1.6) with density function $f=f(x, \xi, t)$ which is a solution to the Vlasov-type equation:

$$
\left\{\begin{array}{l}
\partial_{t} f+\xi \cdot \nabla_{x} f+\nabla_{\xi} \cdot(F[f] f)=0, \\
F[f](x, \xi):=\frac{\int_{\mathbb{R}^{d} \times \mathbb{R}^{d}} \psi^{c s}(|x-y|)\left(\xi_{*}-\xi\right) f\left(y, \xi_{*}\right) d y d \xi_{*}}{\int_{\mathbb{R}^{d}} \psi^{c s}(|x-y|) \rho_{f}(y) d y} \\
f_{0}(x, \xi):=f(x, \xi, 0) .
\end{array}\right.
$$

Now, notice that $F[f]$ can be rewritten as

$$
F[f]=\tilde{u}_{f}-\xi \quad \text { where } \tilde{u}_{f}:=\frac{\int_{\mathbb{R}^{d} \times \mathbb{R}^{d}} \psi^{c s}(|x-y|) \xi_{*} f\left(y, \xi_{*}\right) d y d \xi_{*}}{\int_{\mathbb{R}^{d}} \psi^{c s}(|x-y|) \rho_{f}(y) d y}
$$

and hence that this equation is a non-local version of $(1.1)_{1}$. However, we can localize the previous derivation by assuming that the communication rate is very concentrated around the closest neighbors of a given particle, i.e., that $\psi^{c s}(x)$ is close to a Dirac Delta at the origin. Under this localization of the alignment, it is reasonable to expect (1.1) 1 as the $N \rightarrow \infty$ limit of the Motsch-Tadmor model. Some formal indications on its validity are provided in [23].

In the next section, we state our main results. Then, in Section 3, we provide a priori energy and $L^{p}$ estimates. Section 4 is devoted to the proof of global existence of weak solutions using Schauder's fixed point theorem and velocity averaging lemma. In Section 5, we rigorously investigate the convergence of weak solutions to the system 
(1.1) when the local alignment and diffusive forces are sufficiently strong. In Section 6, we show a priori estimates for long-time behavior of solutions.

Notation. We provide several simplified notations that are used throughout the paper. For a function $f(x, \xi)((x, \xi) \in$ $\mathbb{T}^{3} \times \mathbb{R}^{3}$ ), we denote by $\|f\|_{L^{p}}$ the usual $L^{p}\left(\mathbb{T}^{3} \times \mathbb{R}^{3}\right)$-norm, and if $u$ is a function of $x \in \mathbb{T}^{3}$, we denote by $\|u\|_{L^{p}}$ the usual $L^{p}\left(\mathbb{T}^{3}\right)$-norm, otherwise specified. We also drop $x$-dependence of differential operators $\partial_{x_{i}}, \nabla_{x}$, and $\Delta_{x}$, i.e., $\partial_{i} f:=\partial_{x_{i}} f, \nabla f:=\nabla_{x} f$ and $\Delta f:=\Delta_{x} f$.

\section{Main results}

In this section, we state the three main results of this paper. Our first result concerns the existence of global weak solutions to (1.1). In the second result, we rigorously study a hydrodynamic limit of (1.1) corresponding to strong noise and strong local alignment. Our final result is an estimate on the large-time behavior of solutions to (1.1) with $\sigma=0$. The latter result assumes that the solutions are sufficiently integrable and the particle density is uniformly bounded in time.

\subsection{Existence of weak solutions}

Let us define

$$
\mathcal{H}:=\left\{w \in L^{2}\left(\mathbb{T}^{3}\right) \mid \nabla \cdot w=0\right\}, \quad \mathcal{V}:=\left\{w \in H^{1}\left(\mathbb{T}^{3}\right) \mid \nabla \cdot w=0\right\}
$$

and denote by $\mathcal{V}^{\prime}$ the dual space of $\mathcal{V}$.

Existence will be proved using the following notion of weak solutions.

Definition 2.1. Suppose the initial data $\left(f_{0}, u_{0}\right)$ satisfy

$$
f_{0} \in\left(L_{+}^{1} \cap L^{\infty}\right)\left(\mathbb{T}^{3} \times \mathbb{R}^{3}\right), \quad|\xi|^{2} f_{0} \in L^{1}\left(\mathbb{T}^{3} \times \mathbb{R}^{3}\right), \quad u_{0} \in \mathcal{H}
$$

For a given $T \in(0, \infty)$, we say that the pair $(f, u)$ is a weak solution of (1.1)-(1.2) provided the following conditions are satisfied:

(1) $f \in L^{\infty}\left(0, T ;\left(L_{+}^{1} \cap L^{\infty}\right)\left(\mathbb{T}^{3} \times \mathbb{R}^{3}\right)\right),|\xi|^{2} f \in L^{\infty}\left(0, T ; L^{1}\left(\mathbb{T}^{3} \times \mathbb{R}^{3}\right)\right)$.

(2) $u \in L^{\infty}(0, T ; \mathcal{H}) \cap L^{2}(0, T ; \mathcal{V}) \cap \mathcal{C}^{0}\left([0, T], \mathcal{V}^{\prime}\right)$.

(3) For all $\phi \in \mathcal{C}^{1}\left(\mathbb{T}^{3} \times \mathbb{R}^{3} \times[0, T)\right)$ with $\phi(\cdot, \cdot, T)=0$,

$$
\begin{aligned}
& -\int_{0}^{T} \int_{\mathbb{T}^{3} \times \mathbb{R}^{3}} f\left(\partial_{t} \phi+\xi \cdot \nabla \phi\right) d \xi d x d s \\
& \quad-\int_{0}^{T} \int_{\mathbb{T}^{3} \times \mathbb{R}^{3}}\left(\alpha(u-\xi) f+\beta\left(u_{f}-\xi\right) f-\sigma \nabla_{\xi} f\right) \cdot \nabla_{\xi} \phi d \xi d x d s \\
& =\int_{\mathbb{T}^{3} \times \mathbb{R}^{3}} f_{0} \phi(\cdot, \cdot, 0) d \xi d x .
\end{aligned}
$$

(4) For all $\psi \in\left[\mathcal{C}^{1}\left(\mathbb{T}^{3} \times[0, T]\right)\right]^{3}$, and $\nabla \cdot \psi=0$, for a.e. $t$,

$$
\begin{gathered}
\int_{\mathbb{T}^{3}} u(t) \cdot \psi(t) d x+\int_{0}^{t} \int_{\mathbb{T}^{3}}\left(-u \cdot \partial_{t} \psi-(u \cdot \nabla) \psi \cdot u+\mu \nabla u: \nabla \psi\right) d x d s \\
=-\int_{0}^{t} \int_{\mathbb{T}^{3}}\left(u-u_{f}\right) \cdot \psi \rho_{f} d x d s+\int_{\mathbb{T}^{3}} u_{0} \cdot \psi(0, \cdot) d x .
\end{gathered}
$$


Our existence result is given by the following theorem.

Theorem 2.1. Suppose the initial data $\left(f_{0}, u_{0}\right)$ satisfies (2.1). Then for any $T>0$ there exists at least one weak solution $(f, u)$ to $(1.1)-(1.2)$ on the time-interval $(0, T)$.

The proof of Theorem 2.1 is the topic of Section 4.

\subsection{Hydrodynamic limit}

In our second result, we study the regime where the noise and local alignment are relatively strong compared to the other terms. That is, for $\varepsilon$ small, we consider the system

$$
\begin{aligned}
& \partial_{t} f^{\varepsilon}+\xi \cdot \nabla f^{\varepsilon}+\nabla_{\xi} \cdot\left[\left(u^{\varepsilon}-\xi\right) f^{\varepsilon}\right]=\frac{1}{\varepsilon} \nabla_{\xi} \cdot\left[\nabla_{\xi} f^{\varepsilon}-\left(u_{f^{\varepsilon}}-\xi\right) f^{\varepsilon}\right], \\
& \partial_{t} u^{\varepsilon}+u^{\varepsilon} \cdot \nabla u^{\varepsilon}+\nabla p^{\varepsilon}-\mu \Delta u^{\varepsilon}=-\int_{\mathbb{R}^{3}}\left(u^{\varepsilon}-\xi\right) f^{\varepsilon} d \xi \\
& \nabla \cdot u^{\varepsilon}=0 .
\end{aligned}
$$

Now, observe that the right-hand side can be written

$$
\nabla_{\xi} \cdot\left[\nabla_{\xi} f^{\varepsilon}-\left(u_{f^{\varepsilon}}-\xi\right) f^{\varepsilon}\right]=\nabla_{\xi} \cdot\left(M^{\varepsilon} \nabla_{\xi}\left(\frac{f^{\varepsilon}}{M^{\varepsilon}}\right)\right),
$$

where we have introduced the Maxwellian

$$
M^{\varepsilon}(x, \xi, t):=\frac{1}{(2 \pi)^{3 / 2}} e^{-\frac{\left|\xi-u_{f} \varepsilon(x, t)\right|^{2}}{2}} .
$$

Consequently, if we have that $u_{f} \rightarrow_{f}$ and $u^{\varepsilon} \rightarrow u$, then we expect that $f^{\varepsilon}$ converges to the thermodynamical equilibrium

$$
f^{\varepsilon} \rightarrow M_{\rho_{f}, u_{f}}(x, \xi, t):=\frac{\rho_{f}(x, t)}{(2 \pi)^{3 / 2}} e^{-\frac{\left|\xi-u_{f}(x, t)\right|^{2}}{2}} \text { as } \varepsilon \rightarrow 0 .
$$

In this case, it can be readily seen that $\rho_{f}, u_{f}$, and $u$ evolves according to the fluid-particle model

$$
\begin{aligned}
& \partial_{t} \rho_{f}+\nabla \cdot \rho_{f} u_{f}=0, \\
& \partial_{t}\left(\rho_{f} u_{f}\right)+\nabla \cdot\left(\rho_{f} u_{f} \otimes u_{f}\right)+\nabla \rho_{f}=\rho_{f}\left(u-u_{f}\right), \\
& \partial_{t} u+u \cdot \nabla u+\nabla p-\mu \Delta u=-\rho_{f}\left(u-u_{f}\right), \\
& \nabla \cdot u=0,
\end{aligned}
$$

subject to

$$
\left(\rho_{f}(x, 0), u_{f}(x, 0), u(x, 0)\right)=\left(\rho_{f_{0}}, u_{f_{0}}, u_{0}\right), \quad x \in \mathbb{T}^{3} .
$$

In our second result fact, we prove that weak solutions of (2.2) are close to a strong unique solution of (2.3). Hence, if $\varepsilon$ is sufficiently small, (2.3) provides a good approximation of (2.2).

Theorem 2.2. Assume that there exists a unique strong solution $\left(\rho_{f}, u_{f}, u\right)$ to the system (2.3)-(2.4) in the interval $\left[0, T^{*}\right]$. Furthermore suppose that $\left(f_{0}, u_{0}\right)$ satisfies $(2.1)$, and $f_{0}$ is given by

$$
f_{0}(x, \xi)=\frac{\rho_{f_{0}}(x)}{(2 \pi)^{\frac{3}{2}}} e^{-\left.\frac{\mid \xi-u}{f_{0}}(x)\right|^{2}} .
$$

Then, for any sequences of weak solutions $\left(f^{\varepsilon}, u^{\varepsilon}\right)$ to the system (2.2), we have

$$
\sup _{0 \leq t \leq T^{*}}\left(\left\|u_{f^{\varepsilon}}-u_{f}\right\|_{L^{2}}^{2}+\left\|\rho_{f^{\varepsilon}}-\rho_{f}\right\|_{L^{2}}^{2}+\left\|u^{\varepsilon}-u\right\|_{L^{2}}^{2}\right) \leq C \sqrt{\varepsilon} .
$$


As a consequence, as $\varepsilon \rightarrow 0$,

$$
\begin{aligned}
& f^{\varepsilon} \rightarrow \frac{\rho_{f}}{(2 \pi)^{\frac{3}{2}}} e^{-\frac{\left|\xi-u_{f}\right|^{2}}{2}} \text { in } L_{l o c}^{1}\left(0, T^{*} ; L^{1}\left(\mathbb{T}^{3} \times \mathbb{R}^{3}\right)\right), \\
& \rho_{f^{\varepsilon}} u_{f^{\varepsilon}} \rightarrow \rho_{f} u_{f} \quad \text { in } L_{l o c}^{1}\left(0, T^{*} ; L^{1}\left(\mathbb{T}^{3}\right)\right), \\
& \rho_{f^{\varepsilon}}\left|u_{f^{\varepsilon}}\right|^{2} \rightarrow \rho_{f}\left|u_{f}\right|^{2} \quad \text { in } L_{l o c}^{1}\left(0, T^{*} ; L^{1}\left(\mathbb{T}^{3}\right)\right), \\
& u^{\varepsilon} \rightarrow u \quad \text { in } L_{l o c}^{1}\left(0, T^{*} ; L^{2}\left(\mathbb{T}^{3}\right)\right) .
\end{aligned}
$$

\subsection{Large-time behavior}

Our third result is a large-time behavior estimate for our kinetic model. To state this result, we introduce several energy-fluctuation functions:

$$
\begin{aligned}
& \mathcal{E}_{P}(t):=\frac{1}{2} \int_{\mathbb{T}^{3} \times \mathbb{R}^{3}}\left|\xi-u_{f}\right|^{2} f d x d \xi, \\
& \mathcal{E}_{U}(t):=\frac{1}{2} \int_{\mathbb{T}^{3} \times \mathbb{T}^{3}}\left|u_{f}(x)-u_{f}(y)\right|^{2} \rho_{f}(x) \rho_{f}(y) d x d y, \\
& \mathcal{E}_{F}(t):=\frac{1}{2} \int_{\mathbb{T}^{3}}\left|u-u_{c}(t)\right|^{2} d x, \\
& \mathcal{E}_{I}(t):=\frac{1}{2}\left|u_{c}(t)-\xi_{c}(t)\right|^{2},
\end{aligned}
$$

where $u_{c}$ and $\xi_{c}$ are the mean bulk velocity of the fluid and the averaged particle velocity:

$$
u_{c}:=\int_{\mathbb{T}^{3}} u d x \quad \text { and } \quad \xi_{c}:=\int_{\mathbb{T}^{3} \times \mathbb{R}^{3}} \xi f d \xi d x .
$$

We finally set a total energy function $\mathcal{E}$ :

$$
\mathcal{E}(t):=2 \mathcal{E}_{P}(t)+\mathcal{E}_{U}(t)+2 \mathcal{E}_{F}(t)+\mathcal{E}_{I}(t) .
$$

For this analysis, without loss of generality, we assume $\alpha=\beta=1$.

Theorem 2.3. Let $(f, u)$ be global in time classical solutions to the system (1.1)-(1.2) with $\sigma=0$ satisfying

$$
\mathcal{E}(0)<\infty, \quad \lim _{|\xi| \rightarrow \infty}|\xi|^{2} f(x, \xi, t)=0, \quad(x, t) \in \mathbb{T}^{3} \times[0, \infty) .
$$

Assume that $\left\|\rho_{f}\right\|_{L^{\infty}\left(0, \infty ; L^{3 / 2}\left(\mathbb{T}^{3}\right)\right)}<\infty$, then the total energy fluctuation function $\mathcal{E}(t)$ satisfies

$$
\frac{d}{d t} \mathcal{E}(t) \leq-C \mathcal{E}(t), \quad \text { for } t \in[0, \infty),
$$

where $C$ is a positive constant depending on $\mu, \rho_{f}$.

Remark 2.1. Since the total momentum $u_{c}(t)+\xi_{c}(t)$ is conserved, we find

$$
\frac{1}{2} \mathcal{E}_{I}(t)=\left|u_{c}(t)-\frac{1}{2}\left(\xi_{c}(0)+u_{c}(0)\right)\right|^{2}=\left|\xi_{c}(t)-\frac{1}{2}\left(\xi_{c}(0)+u_{c}(0)\right)\right|^{2} .
$$

Thus this deduces the emergence of exponential alignment between particles and fluid, and they asymptotically converge to half of the initial total momentum. Notice that the previous theorem makes no assumption on the viscosity of the fluid. 


\section{Preliminary material}

The purpose of this section is to derive a priori energy and $L^{p}$ estimates for the system (1.1). We will also provide two technical lemmata that will be frequently applied in the subsequent analysis. In this process, we shall use the following notations for the $k$-th local and global momentums

$$
m_{k}(f)(x, t)=\int_{\mathbb{R}^{3}}|\xi|^{k} f d \xi, \quad M_{k}(f)(t):=\int_{\mathbb{T}^{3} \times \mathbb{R}^{3}}|\xi|^{k} f(x, \xi) d x d \xi,
$$

where $k=0,1 \ldots$ We also observe that

$$
\rho_{f}=m_{0}(f)(x, t), \quad\left|\rho_{f} u_{f}\right| \leq m_{1}(f)(x, t), \quad \text { and } \quad M_{k}(f)(t)=\int_{\mathbb{T}^{3}} m_{k}(f) d x .
$$

\subsection{A priori energy and $L^{p}$ estimate}

The following proposition provides an energy estimate.

Proposition 3.1. Let $(f, u)$ be any fast decaying at infinity smooth solutions to the system (1.1). Then, the following properties hold

$$
\begin{aligned}
& \text { (i) } \frac{d}{d t} \int_{\mathbb{T}^{3} \times \mathbb{R}^{3}} f d \xi d x=0, \\
& \text { (ii) } \frac{d}{d t}\left(\int_{\mathbb{T}^{3}} u d x+\int_{\mathbb{T}^{3} \times \mathbb{R}^{3}} \xi f d \xi d x\right)=0 \\
& \text { (iii) } \frac{1}{2} \frac{d}{d t}\left(\int_{\mathbb{T}^{3} \times \mathbb{R}^{3}}|\xi|^{2} f d \xi d x+\int_{\mathbb{T}^{3}}|u|^{2} d x\right)+\mu \int_{\mathbb{T}^{3}}|\nabla u|^{2} d x \\
& =-\alpha \int_{\mathbb{T}^{3} \times \mathbb{R}^{3}}|u-\xi|^{2} f d \xi d x-\beta \int_{\mathbb{T}^{3} \times \mathbb{R}^{3}}\left|u_{f}-\xi\right|^{2} f d \xi d x+3 \sigma \int_{\mathbb{T}^{3} \times \mathbb{R}^{3}} f d \xi d x .
\end{aligned}
$$

Proof. (i) and (ii) are readily obtained from the system (1.1). For the estimate of (iii), we multiply (1.1) 1 by $|\xi|^{2} / 2$ and integrating over $\mathbb{T}^{3} \times \mathbb{R}^{3}$ to get

$$
\begin{aligned}
& \frac{1}{2} \frac{d}{d t} \int_{\mathbb{T}^{3} \times \mathbb{R}^{3}}|\xi|^{2} f d \xi d x \\
& =\alpha \int_{\mathbb{T}^{3} \times \mathbb{R}^{3}} \xi \cdot(u-\xi) f d \xi d x+\beta \int_{\mathbb{T}^{3} \times \mathbb{R}^{3}}\left|u_{f}-\xi\right|^{2} f d \xi d x \\
& \quad+3 \sigma \int_{\mathbb{T}^{3} \times \mathbb{R}^{3}} f d \xi d x,
\end{aligned}
$$

where we have used that

$$
\int_{\mathbb{T}^{3} \times \mathbb{R}^{3}} u_{f} \cdot\left(u_{f}-\xi\right) f d \xi d x=0
$$


On the other hand, from $(1.1)_{3}$, we get

$$
\frac{1}{2} \frac{d}{d t} \int_{\mathbb{T}^{3}}|u|^{2} d x+\mu \int_{\mathbb{T}^{3}}|\nabla u|^{2} d x=-\alpha \int_{\mathbb{T}^{3} \times \mathbb{R}^{3}} u \cdot(u-\xi) f d \xi d x .
$$

We now combine (3.1) and (3.2) to conclude the desired result.

Remark 3.1. Throughout the paper, without loss of generality, we assume

$$
M_{0}\left(f_{0}\right)=\int_{\mathbb{T}^{3} \times \mathbb{R}^{3}} f_{0}(x, \xi) d x d \xi=1 .
$$

Then it follows from mass conservation (Proposition 3.1(i)) that

$$
M_{0}(f)(t)=\int_{\mathbb{T}^{3} \times \mathbb{R}^{3}} f(x, \xi, t) d x d \xi=1, \quad t \geq 0 .
$$

We next provide an $L^{p}$-estimate for the particle density function $f$.

Proposition 3.2. Let $(f, u)$ be any smooth solutions to the system (1.1). Then we have

$$
\frac{d}{d t}\|f\|_{L^{p}}^{p}+\frac{4 \sigma(p-1)}{p}\left\|\nabla_{\xi} f^{\frac{p}{2}}\right\|_{L^{2}}^{2}=3(\alpha+\beta)(p-1)\|f\|_{L^{p}}^{p} .
$$

In particular, we have that

$$
\|f\|_{L^{\infty}\left(\mathbb{T}^{3} \times \mathbb{R}^{3} \times[0, T]\right)} \leq C(T, \alpha, \beta)\left\|f_{0}\right\|_{L^{\infty}\left(\mathbb{T}^{3} \times \mathbb{R}^{3}\right)} .
$$

Proof. (i) Multiplying (1.1) $)_{1}$ by $p f^{p-1}$ and integrate over $\mathbb{T}^{3} \times \mathbb{R}^{3}$ to obtain

$$
\begin{aligned}
\frac{d}{d t} & \int_{\mathbb{T}^{3} \times \mathbb{R}^{3}} f^{p} d x d \xi \\
= & -\alpha p \int_{\mathbb{T}^{3} \times \mathbb{R}^{3}} f^{p-1} \nabla_{\xi} \cdot((u-\xi) f) d x d \xi \\
& -\beta p \int_{\mathbb{T}^{3} \times \mathbb{R}^{3}} f^{p-1} \nabla_{\xi} \cdot\left(\left(u_{f}-\xi\right) f\right) d x d \xi+\sigma p \int_{\mathbb{T}^{3} \times \mathbb{R}^{3}} \Delta_{\xi} f d x d \xi \\
= & : I_{1}+I_{2}+I_{3} .
\end{aligned}
$$

For the estimates of $I_{i}, i=1,2,3$, it is straightforward to get by integration by parts

$$
\begin{aligned}
& I_{1}=3 \alpha(p-1) \int_{\mathbb{T}^{3} \times \mathbb{R}^{3}} f^{p} d x d \xi, \\
& I_{2}=3 \beta(p-1) \int_{\mathbb{T}^{3} \times \mathbb{R}^{3}} f^{p} d x d \xi, \\
& I_{3}=-\frac{4 \sigma(p-1)}{p} \int_{\mathbb{T}^{3} \times \mathbb{R}^{3}}\left|\nabla_{\xi} f^{\frac{p}{2}}\right|^{2} d x d \xi .
\end{aligned}
$$

This concludes the proof. 


\subsection{Integrability and velocity averaging}

Let us now provide two useful lemmas for later reference. For the proofs of these lemmas, we refer to [3,13,21].

Lemma 3.1. Let $k_{2}>k_{1}$ and $f$ be a nonnegative function. Suppose $f$ satisfies

$$
\|f\|_{L^{\infty}\left(\mathbb{T}^{3} \times \mathbb{R}^{3} \times[0, T]\right)}<\infty, \quad \text { and } m_{k_{2}}(f)(x, t)<\infty, \quad \text { a.e. }(x, t) .
$$

Then the following inequality holds

$$
m_{k_{1}}(f)(x, t) \leq\left(\frac{4 \pi}{3}\|f\|_{L^{\infty}\left(\mathbb{T}^{3} \times \mathbb{R}^{3} \times[0, T]\right)}+1\right) m_{k_{2}}(f)(x, t)^{\frac{k_{1}+3}{k_{2}+3}}, \quad \text { a.e. }(x, t) .
$$

We conclude this section by stating the following version of the celebrated velocity averaging lemma.

Lemma 3.2. For $1 \leq p<\frac{5}{4}$, let $\left\{G^{n}\right\}_{n}$ be bounded in $L^{p}\left(\mathbb{T}^{3} \times \mathbb{R}^{3} \times(0, T)\right)$. Suppose that

$f^{n}$ is bounded in $L^{\infty}\left(0, T ; L^{1} \cap L^{\infty}\left(\mathbb{T}^{3} \times \mathbb{R}^{3}\right)\right)$,

$|\xi|^{2} f^{n}$ is bounded in $L^{\infty}\left(0, T ; L^{1}\left(\mathbb{T}^{3} \times \mathbb{R}^{3}\right)\right)$.

If $f^{n}$ and $G^{n}$ satisfy the equation

$$
f_{t}^{n}+\xi \cdot \nabla f^{n}=\nabla_{\xi}^{k} G^{n},\left.\quad f^{n}\right|_{t=0}=f_{0} \in L^{p}\left(\mathbb{T}^{3} \times \mathbb{R}^{3}\right),
$$

for a multi-index $k$. Then, for any $\psi(\xi)$, such that $|\psi(\xi)| \leq c|\xi|$ as $|\xi| \rightarrow \infty$, the sequence

$$
\left\{\int_{\mathbb{R}^{3}} f^{n} \psi(\xi) d \xi\right\}_{n}
$$

is relatively compact in $L^{p}\left(\mathbb{T}^{3} \times(0, T)\right)$.

\section{Global existence of weak solutions (Theorem 2.1)}

In this section, we will prove the existence of weak solutions to the system (1.1) and thereby prove Theorem 2.1. Our strategy will be to pass to the limit in a sequence of approximate solutions. To define the approximate solutions, fix a small $\varepsilon>0$, let $\theta$ be a standard mollifier:

$$
\theta \geq 0, \quad \theta \in \mathcal{C}_{0}^{\infty}\left(\mathbb{T}^{3}\right), \quad \operatorname{supp}_{x} \theta \subset B_{1}(0), \quad \int_{\mathbb{T}^{3}} \theta(x) d x=1,
$$

and set $\theta_{\varepsilon}(x):=\left(1 / \varepsilon^{3}\right) \theta(x / \varepsilon)$. The approximate solutions are obtained by solving:

$$
\begin{aligned}
& \partial_{t} f+\xi \cdot \nabla f+\nabla_{\xi} \cdot\left[f\left(\chi_{R}(u)-\xi\right)\right]=-\nabla_{\xi} \cdot\left[f\left(\chi_{R}\left(u_{f}^{\varepsilon}\right)-\xi\right)\right]+\sigma \Delta_{\xi} f, \\
& \partial_{t} u+\left(\theta_{\varepsilon} \star u\right) \cdot \nabla u+\nabla p=\mu \Delta u+\left(m_{f}-\varrho_{f} u\right) \mathbf{1}_{R}(u), \\
& \nabla \cdot u=0
\end{aligned}
$$

where $m_{f}=\int_{\mathbb{R}^{3}} \xi f d \xi$. Compared to (1.1), we have introduced the regularizations

$$
\mathbf{1}_{R}(w)=\left\{\begin{array}{ll}
1, & |w| \leq R, \\
0, & \text { otherwise }
\end{array}, \quad \chi_{R}(w)=w \mathbf{1}_{R}(w), \quad \text { and } \quad u_{f}^{\varepsilon}=\frac{m_{f}}{\varrho_{f}+\varepsilon},\right.
$$

and in addition, we have regularized the convection velocity $\theta_{\varepsilon} \star u$. Notice that we do not need the notation of $u_{f}$.

We shall also need to regularize the initial data:

$$
u_{0}^{\varepsilon}:=\theta_{\varepsilon} \star u_{0}, \quad f_{0}^{R}:=f_{0} \mathbf{1}_{R}(\xi) .
$$


Remark 4.1. In our approximation scheme (4.1), for simplicity, we set $\alpha=\beta=1$. We also dropped the subscript $\varepsilon$ and $R$, for instance $f^{\varepsilon, R}$ or $u^{\varepsilon, R}$ by $f$ or $u$.

Before we can start sending $\varepsilon \rightarrow 0$ and $R \rightarrow \infty$ in (4.1), we need to make sure that (4.1) actually admits a weak solution. We will establish the following proposition.

Proposition 4.1. For a given $T>0$, suppose that $\left(f_{0}, u_{0}\right)$ satisfy (4.3). Then there exists a weak solution $(f, u)$ to (4.1) in the sense of Definition 2.1 (with $\chi_{R}\left(u_{f}^{\varepsilon}\right)$ replacing $u_{f}$ ).

For the proof of Proposition 4.1, we will consider another decoupled system which is defined in the next subsection.

\subsection{The regularized and linearized system}

We shall prove Proposition 4.1 using a fixed point argument. For this purpose, we will use the space

$$
\mathcal{S}:=L^{2}\left(\mathbb{T}^{3} \times(0, T)\right) \times L^{2}\left(\mathbb{T}^{3} \times(0, T)\right) .
$$

For $(w, \bar{u}) \in \mathcal{S}$ given, let $(f, u)$ be a weak solution to

$$
\begin{aligned}
& \partial_{t} f+\xi \cdot \nabla f+\nabla_{\xi} \cdot\left[f\left(\chi_{R}(w)-\xi\right)\right]=-\nabla_{\xi} \cdot\left[f\left(\chi_{R}(\bar{u})-\xi\right)\right]+\sigma \Delta_{\xi} f, \\
& \partial_{t} u+\left(\theta_{\varepsilon} \star u\right) \cdot \nabla u+\nabla p=\mu \Delta u+\left(m_{f}-\varrho_{f} w\right) \mathbf{1}_{R}(w), \\
& \nabla \cdot u=0
\end{aligned}
$$

and define the operator $\mathcal{T}: \mathcal{S} \mapsto \mathcal{S}$ through the relation

$$
\mathcal{T}[w, \bar{u}]:=\left[u, u_{f}^{\varepsilon}\right]=\left[u, \frac{m_{f}}{\varepsilon+\varrho_{f}}\right] .
$$

Observe that a fixed point $\left[u, u_{f}^{\varepsilon}\right]=\mathcal{T}\left[u, u_{f}^{\varepsilon}\right]$ is also a solution of (4.1). Hence, Proposition 4.1 follows if we are able to establish the existence of such a fixed point. In this subsection, we shall achieve this by verifying the postulates of the Schauder fixed point theorem.

\subsubsection{The operator $\mathcal{T}[\cdot, \cdot]$ is well-defined}

Lemma 4.1. Let $\left(f_{0}, u_{0}\right)$ satisfy (4.3), and assume that we are given $(w, \bar{u}) \in \mathcal{S}$. Then there exists a unique solution $(f, u)$ of (4.4) satisfying

$$
\begin{aligned}
\|f\|_{L^{\infty}\left(0, T ; L^{p}\left(\mathbb{T}^{3} \times \mathbb{R}^{3}\right)\right)}+\left\|\nabla_{\xi} f^{\frac{p}{2}}\right\|_{L^{2}\left(0, T ; L^{2}\left(\mathbb{T}^{3} \times \mathbb{R}^{3}\right)\right)}^{\frac{2}{p}} & \leq C(R, \sigma, T)\left\|f_{0}\right\|_{L^{p}\left(\mathbb{T}^{3} \times \mathbb{R}^{3}\right),} \\
\sup _{t \in(0, T)} \int f|\xi|^{k} d \xi d x & \leq C(R, k, \sigma, T),
\end{aligned}
$$

for $p \in[1, \infty]$ and all finite $k$, and moreover,

$$
\frac{1}{2}\|u\|_{L^{\infty}\left(0, T ; L^{2}\left(\mathbb{T}^{3}\right)\right)}+\mu\|\nabla u\|_{L^{2}\left(0, T ; L^{2}\left(\mathbb{T}^{3}\right)\right)} \leq \frac{1}{2}\left\|u_{0}\right\|_{L^{\infty}\left(0, T ; L^{2}\left(\mathbb{T}^{3}\right)\right)}+C(R, T) .
$$

Here, $C(\cdot)$ denotes a generic constant depending on $\cdot$

Proof. First, we observe that the two equations in (4.4) are decoupled and a solution can be obtained by first determining $f$ and then $u$. Let us begin by discussing solutions to the first equation.

1. Since both $\chi_{R}(w)$ and $\chi_{R}(\bar{u})$ are bounded in $L^{\infty}\left(\mathbb{T}^{3} \times(0, T)\right)$, existence of a unique function $f \in$ $\mathcal{C}\left([0, T] ;\left(L^{1} \cap L^{\infty}\right)\left(\mathbb{T}^{3} \times \mathbb{R}^{3}\right)\right)$ solving (4.4) is by now standard and can be found in [11] (cf. [21]). The $L^{p}$ bound in (4.5) can be found in [21]. We also notice that for a smooth solution $f$ to (4.4) provides 


$$
\begin{aligned}
\frac{d}{d t} \int_{\mathbb{T}^{3} \times \mathbb{R}^{3}} f|\xi|^{k} d \xi d x & \leq \int_{\mathbb{T}^{3} \times \mathbb{R}^{3}} f\left(2 k R|\xi|^{k-1}+\sigma k(k+1)|\xi|^{k-2}\right) d \xi d x \\
& \leq C(R, k, \sigma)\left(\int_{\mathbb{T}^{3} \times \mathbb{R}^{3}} f_{0} d \xi d x+\int_{\mathbb{T}^{3} \times \mathbb{R}^{3}} f|\xi|^{k} d \xi d x\right) .
\end{aligned}
$$

Since $\int_{\mathbb{T}^{3} \times \mathbb{R}^{3}} f_{0} d \xi d x<\infty$ and $\int_{\mathbb{T}^{3} \times \mathbb{R}^{3}} f_{0}^{R}|\xi|^{k} d \xi d x<\infty$ for any finite $k$, we obtain that

$$
\sup _{t \in(0, T)} \int_{\mathbb{T}^{3} \times \mathbb{R}^{3}} f|\xi|^{k} d \xi d x<C(R, k, \sigma, T) \quad \text { for any } k \text { finite. }
$$

This bound continues to hold for the unique solution $f$ of (4.4). To see this, one can for instance localize $|\xi|^{k}$ as $\phi\left(|\xi|^{k}\right)$ where $\phi(r)=1$ when $r \leq D$, and $\phi=0$ when $r \geq 2 D$, make the corresponding calculations and send $D \rightarrow \infty$. This concludes the second inequality in (4.5).

2. Let us now turn to the Navier-Stokes equations for $u$. First, since all (finite) moments of $f$ are bounded (4.7), Lemma 3.1 gives in particular

$$
\varrho_{f}, m_{f} \in L^{\infty}\left(0, T ; L^{2}\left(\mathbb{T}^{3}\right)\right),
$$

where the inclusion constant depends on $R$. Due to (4.8), we see that the right-hand side in the equation for $u$ is also in $L^{\infty}\left(0, T ; L^{2}\left(\mathbb{T}^{3}\right)\right)$, that is,

$$
\left\|\left(m_{f}-\varrho_{f} w\right) \mathbf{1}_{|w| \leq R}\right\|_{L^{\infty}\left(0, T ; L^{2}\left(\mathbb{T}^{3}\right)\right)} \leq C(R) .
$$

Standard parabolic theory then asserts the existence of a unique solution $u$ satisfying (4.6) (cf. [12]).

From the previous lemma, it readily follows that $\mathcal{T}[\cdot, \cdot]$ is well-defined and maps into a bounded subset of $\mathcal{S}$.

Corollary 4.1. There is a constant $C(R, \varepsilon)$, such that

$$
\|\mathcal{T}[w, \bar{u}]\|_{\mathcal{S}} \leq C(R, \varepsilon), \quad \forall(w, \bar{u}) \in \mathcal{S}
$$

Proof. By definition, we have that

$$
\begin{aligned}
\|\mathcal{T}[w, \bar{u}]\|_{S} & \leq\|u\|_{L^{2}\left(\mathbb{T}^{3} \times(0, T)\right)}+\frac{1}{\varepsilon}\left\|m_{f}\right\|_{L^{2}\left(\mathbb{T}^{3} \times(0, T)\right)} \\
& \leq C(R, \varepsilon, T),
\end{aligned}
$$

where the last inequality is (4.5) and (4.6).

\subsubsection{The operator $\mathcal{T}[\cdot, \cdot]$ is compact}

Lemma 4.2. Let $\left(f_{0}, u_{0}\right)$ and $T$ be as in Proposition 4.1, and let $\left\{\left(w_{n}, \bar{u}_{n}\right)\right\}_{n=0}^{\infty}$ be a uniformly bounded sequence in $\mathcal{S}$. Then up to a subsequence $\left\{\mathcal{T}\left[w_{n}, \bar{u}_{n}\right]\right\}_{n=0}^{\infty}$ converges strongly in $\mathcal{S}$.

Proof. Let $\left\{\left(u_{n}, f_{n}\right)\right\}_{n}$ be the sequence of solutions to (4.4) corresponding to $\left\{\left(w_{n}, \bar{u}_{n}\right)\right\}_{n}$. We will prove compactness of the two components of $\mathcal{T}[\cdot, \cdot]$ separately.

1. We take the first component of $\mathcal{T}\left[w_{n}, \bar{u}_{n}\right],\left.\mathcal{T}\left[w_{n}, \bar{u}_{n}\right]\right|_{1}=u_{n}$. To show its compactness in $L^{2}\left(0, T ; L^{2}\left(\mathbb{T}^{3}\right)\right)$, it suffices to prove that

$$
\left\|u_{n}\right\|_{L^{2}\left(0, T ; H^{1}\right)} \leq C, \quad \text { and } \quad\left\|\partial_{t} u_{n}\right\|_{L^{2}\left(0, T ; \mathcal{V}^{\prime}\right)} \leq C,
$$

due to the Aubin-Lions compactness lemma. 
- Estimate of $\left\|u_{n}\right\|_{L^{2}\left(0, T ; H^{1}\right)} \leq C$ : From (4.4), we get

$$
\begin{aligned}
\frac{1}{2} \frac{d}{d t} \int_{\mathbb{T}^{3}}\left|u_{n}\right|^{2} d x+\mu \int_{\mathbb{T}^{3}}\left|\nabla u_{n}\right|^{2} d x & =-\int_{\mathbb{T}^{3} \times \mathbb{R}^{3}}(w-\xi) f_{n} \mathbf{1}_{|w| \leq R} \cdot u_{n} d \xi d x \\
& \leq\left\|\rho_{f_{n}}\right\|_{L^{2}}\left\|u_{n}\right\|_{L^{2}}+\left\|m_{f_{n}}\right\|_{L^{2}}\left\|u_{n}\right\|_{L^{2}} \\
& \leq\left(\left\|\rho_{f_{n}}\right\|_{L^{2}}+\left\|m_{f_{n}}\right\|_{L^{2}}\right)^{2}+\left\|u_{n}\right\|_{L^{2}}^{2} .
\end{aligned}
$$

Then it follows from Lemma 3.1 that

$$
\frac{1}{2} \frac{d}{d t}\left\|u_{n}\right\|_{L^{2}}^{2}+\mu\left\|\nabla u_{n}\right\|_{L^{2}}^{2} \leq C+\left\|u_{n}\right\|_{L^{2}}^{2},
$$

and this yields

$$
\left\|u_{n}\right\|_{L^{\infty}\left(0, T ; L^{2}\right)} \leq C, \quad \text { and } \quad\left\|\nabla u_{n}\right\|_{L^{2}\left(0, T ; L^{2}\right)} \leq C .
$$

- Estimate of $\left\|\partial_{t} u_{n}\right\|_{L^{2}\left(0, T ; \mathcal{V}^{\prime}\right)} \leq C$ : For this, it is enough to check the convection and drag force terms. For $\phi \in \mathcal{V}$, we obtain

$$
\begin{aligned}
\left|\int_{0}^{T} \int_{\mathbb{T}^{3}}\left(\left(\theta_{\varepsilon} \star u_{n}\right) \cdot \nabla u_{n}\right) \cdot \phi d x d t\right| & =\left|\int_{0}^{T} \int_{\mathbb{T}^{3}}\left(\left(\theta_{\varepsilon} \star u_{n}\right) \cdot \nabla \phi\right) \cdot u_{n} d x d t\right| \\
& \leq \int_{0}^{T}\left\|\theta_{\varepsilon} \star u_{n}\right\|_{L^{\infty}}\|\nabla \phi\|_{L^{2}}\left\|u_{n}\right\|_{L^{2}} d t \\
& \leq\left\|\theta_{\varepsilon}\right\|_{L^{2}}\left\|u_{n}\right\|_{L^{\infty}\left(0, T ; L^{2}\right)}^{2} \int_{0}^{T}\|\nabla \phi\|_{L^{2}} d t \\
& \leq C(T, \varepsilon)\|\nabla \phi\|_{L^{2}\left(0, T ; L^{2}\right)},
\end{aligned}
$$

by (4.9). This implies

$$
\phi \mapsto \int_{0}^{T} \int_{\mathbb{T}^{3}}\left(\left(\theta_{\varepsilon} \star u_{n}\right) \cdot \nabla u_{n}\right) \cdot \phi d x d t \quad \text { is bounded in } L^{2}\left(0, T ; \mathcal{V}^{\prime}\right) .
$$

For the drag force term, we obtain

$$
\begin{aligned}
& \left|\int_{0}^{T} \int_{\mathbb{T}^{3} \times \mathbb{R}^{3}}(w-\xi) f_{n} \mathbf{1}_{|w| \leq R} \cdot \phi d \xi d x d t\right| \\
& \quad \leq R\|\phi\|_{L^{2}\left(0, T ; L^{2}\right)}\left\|\rho_{f_{n}}\right\|_{L^{2}\left(0, T ; L^{2}\right)}+\|\phi\|_{L^{2}\left(0, T ; L^{5}\right)}\left\|m_{f_{n}}\right\|_{L^{2}\left(0, T ; L^{\frac{5}{4}}\right)} \\
& \quad \leq C\|\phi\|_{L^{2}\left(0, T ; H^{1}\right)} .
\end{aligned}
$$

Here we used again Lemma 3.1 and $\mathbb{T}^{3}$ is bounded. Hence we conclude that the drag force term is uniformly bounded in $L^{2}\left(0, T ; \mathcal{V}^{\prime}\right)$.

2. The second component of $\mathcal{T}[\cdot, \cdot]$ is given by

$$
\left.\mathcal{T}\left[w_{n}, \bar{u}_{n}\right]\right|_{2}=\frac{m_{f_{n}}}{\varepsilon+\varrho_{f_{n}}},
$$

and hence strong convergence follows if we can prove the compactness of $\varrho_{f_{n}}$ and $m_{f_{n}}$. From (4.8), we have that

$$
\varrho_{f_{n}}, m_{f_{n}} \in \in_{b} L^{2}\left(\mathbb{T}^{3} \times(0, T)\right),
$$


where $\epsilon_{b}$ means that the inclusion constant is independent of $n$. To show the compactness, we write (4.4) in the form

$$
\partial_{t} f_{n}+\xi \cdot \nabla f_{n}=\nabla_{\xi} \cdot G_{n}+\sigma \Delta_{\xi} f_{n},
$$

where we have introduced the quantity

$$
G_{n}=f_{n}\left(\chi_{R}\left(w_{n}\right)+\chi_{R}\left(\bar{u}_{n}\right)-2 \xi\right) .
$$

For any finite $2 \leq p<\infty$, an application of the Hölder inequality provides

$$
\begin{aligned}
& \left\|G_{n}\right\|_{L^{p}\left(\mathbb{T}^{3} \times R^{3}\right)} \\
& \quad \leq C(R)\left\|f_{n}\right\|_{L^{p}\left(\mathbb{T}^{3} \times \mathbb{R}^{3}\right)}+2\left\|f_{n}\right\|_{L^{\infty}\left(\mathbb{T}^{3} \times \mathbb{R}^{3}\right)}^{\frac{p-1}{p}}\left(\int_{\mathbb{T}^{3} \times \mathbb{R}^{3}}\left|f_{n} \| \xi\right|^{p} d \xi d x\right)^{\frac{1}{p}} \leq C(R),
\end{aligned}
$$

where the last inequality is (4.5)-(4.6). Hence, we can conclude that

$$
G_{n} \in_{b} L^{p}\left(\mathbb{T}^{3} \times \mathbb{R}^{3} \times(0, T)\right) \text { for all } p \in(1, \infty) .
$$

The velocity averaging Lemma 3.2 is then applicable and yields that $\left\{\varrho_{f_{n}}\right\}_{n}$ and $\left\{m_{f_{n}}\right\}_{n}$ are relatively compact in $L^{2}\left(\mathbb{T}^{3} \times(0, T)\right)$. This concludes the proof of compactness of the operator $\mathcal{T}$.

Proof of Proposition 4.1. Through Lemma 4.1, Corollary 4.1, and Lemma 4.2, we have established that the operator $\mathcal{T}[\cdot, \cdot]$ is well-defined, bounded, and compact. Moreover, continuity of the operator $\mathcal{T}[\cdot, \cdot \cdot]$ is straightforward. As a consequence, the postulates of the Schauder fixed point theorem are satisfied, and hence yields the existence of a fixed point. This concludes our proof of Proposition 4.1.

\subsection{Uniform bounds}

To consider vanishing approximation parameters, we will need some uniform (in $\varepsilon$ and $R$ ) $L^{p}$ and energy bounds on solutions of (4.1). We recall that the energy is given by

$$
\mathbb{E}(t)=\int_{\mathbb{T}^{3} \times \mathbb{R}^{3}} f \frac{|\xi|^{2}}{2} d \xi d x+\int_{\mathbb{T}^{3}} \frac{|u|^{2}}{2} d x .
$$

Lemma 4.3. Under the conditions of Proposition 4.1, there exists a constant $C>0$, independent of $R$ and $\varepsilon$, such that

$$
\begin{aligned}
& \|f\|_{L^{\infty}\left(0, T ; L^{p}\left(\mathbb{T}^{3} \times \mathbb{R}^{3}\right)\right)}+\left\|\nabla_{\xi} f^{\frac{p}{2}}\right\|_{L^{2}\left(\mathbb{T}^{3} \times \mathbb{R}^{3} \times(0, T)\right)}^{\frac{2}{p}} \leq C(p, \sigma, T)\left\|f_{0}\right\|_{L^{p}\left(\mathbb{T}^{3} \times \mathbb{R}^{3}\right)}, \\
& \sup _{t \in(0, T)} \mathbb{E}(t)+\mu \int_{0}^{T}\|\nabla u\|_{L^{2}\left(\mathbb{T}^{3}\right)}^{2} d t+\int_{\mathbb{T}^{3} \times \mathbb{R}^{3}} f\left|\chi_{R}(u)-\xi\right|^{2} d \xi d x \\
& \quad \leq \mathbb{E}(0)+3 \sigma M_{0}\left(f_{0}\right) T .
\end{aligned}
$$

Proof. By direct calculation using (4.1), we deduce

$$
\begin{aligned}
\frac{d}{d t} & \|f\|_{L^{p}\left(\mathbb{T}^{3} \times \mathbb{R}^{3}\right)}^{p}+\frac{4 \sigma(p-1)}{p}\left\|\nabla_{\xi} f^{\frac{p}{2}}\right\|_{L^{2}\left(\mathbb{T}^{3} \times \mathbb{R}^{3}\right)}^{2} \\
& =\int_{\mathbb{T}^{3} \times \mathbb{R}^{3}}\left(\chi_{R}(u)+\chi_{R}\left(u_{f}^{\varepsilon}\right)-2 \xi\right) \cdot \nabla_{\xi} f^{p} d \xi d x=6\|f\|_{L^{p}\left(\mathbb{T}^{3} \times \mathbb{R}^{3}\right)}^{p}
\end{aligned}
$$

and (4.10) follows from the Gronwall inequality. 
Next, we calculate $\mathbb{E}^{\prime}(t)$ using both equations in (4.1);

$$
\begin{aligned}
\mathbb{E}^{\prime}= & \int_{\mathbb{T}^{3}} u_{t} \cdot u d x+\int_{\mathbb{T}^{3} \times \mathbb{R}^{3}} f_{t} \frac{|\xi|^{2}}{2} d \xi d x \\
= & -\mu\|\nabla u\|_{L^{2}\left(\mathbb{T}^{3}\right)}^{2}-\int_{\mathbb{T}^{3}}\left(\left(\theta_{\varepsilon} \star u\right) \cdot \nabla u\right) \cdot u d x+\int_{\mathbb{T}^{3}}\left(m_{f}-\varrho_{f} u\right) \mathbf{1}_{R}(u) \cdot u d x \\
& +3 \sigma M_{0}\left(f_{0}\right)+\int_{\mathbb{T}^{3} \times \mathbb{R}^{3}} f\left(\chi_{R}(u)-\xi\right) \cdot \xi d \xi d x \\
& +\int_{\mathbb{T}^{3} \times \mathbb{R}^{3}} f\left(\chi_{R}\left(u_{f}^{\varepsilon}\right)-\xi\right) \cdot \xi d \xi d x .
\end{aligned}
$$

By adding and subtracting, we deduce that

$$
\begin{aligned}
& \int_{\mathbb{T}^{3} \times \mathbb{R}^{3}} f\left(\chi_{R}(u)-\xi\right) \cdot \xi d \xi d x \\
& =-\int_{\mathbb{T}^{3} \times \mathbb{R}^{3}} f\left|\chi_{R}(u)-\xi\right|^{2} d \xi d x+\int_{\mathbb{T}^{3}}\left(\varrho_{f} \chi_{R}(u)-m_{f}\right) \cdot \chi_{R}(u) d x \\
& =-\int_{\mathbb{T}^{3} \times \mathbb{R}^{3}} f\left|\chi_{R}(u)-\xi\right|^{2} d \xi d x+\int_{\mathbb{T}^{3}}\left(\varrho_{f} u-m_{f}\right) \mathbf{1}_{R}(u) \cdot u d x .
\end{aligned}
$$

We also have

$$
\int_{\mathbb{T}^{3} \times \mathbb{R}^{3}} f\left(\chi_{R}\left(u_{f}^{\varepsilon}\right)-\xi\right) \cdot \xi d \xi d x \leq \int_{\mathbb{T}^{3}} \frac{\left|m_{f}\right|^{2}}{\rho_{f}+\varepsilon} d x-\int_{\mathbb{T}^{3} \times \mathbb{R}^{3}}|\xi|^{2} f d \xi d x \leq 0,
$$

where we used $\left|m_{f}\right|^{2} \leq \rho_{f}\left(\int_{\mathbb{R}^{3}}|\xi|^{2} f d \xi\right)$. By applying (4.13) and (4.14) in (4.12), we obtain (4.11).

\subsection{The $R \rightarrow \infty$ limit}

We are now ready to send $R \rightarrow \infty$ in our approximate equation (4.1). We begin by deriving some compactness properties. Some of the arguments we shall use in this regard are similar to those of [21].

Lemma 4.4. Let $\varepsilon>0$ be fixed, set $R=n$, and let $\left\{\left(f_{n}, u_{n}\right)\right\}_{n=0}^{\infty}$ be the corresponding sequence of solutions to (4.1). Then, up to a subsequence as $n \rightarrow \infty$, we have

$$
\begin{aligned}
& f_{n} \rightarrow f \quad \text { in } \mathcal{C}\left([0, T] ; L^{p}\left(\mathbb{T}^{3} \times \mathbb{R}^{3}\right)\right), p \in(1, \infty), \\
& \varrho_{f_{n}} \rightarrow \varrho_{f} \quad \text { a.e. and in } L^{p}\left(\mathbb{T}^{3} \times(0, T)\right), p \in\left(1, \frac{5}{4}\right), \\
& m_{f_{n}} \rightarrow m_{f} \quad \text { a.e. and in } L^{q}\left(\mathbb{T}^{3} \times(0, T)\right), q \in\left(1, \frac{5}{4}\right), \\
& u_{n} \rightarrow u \quad \text { a.e. and in } L^{2}\left(\mathbb{T}^{3} \times(0, T)\right),
\end{aligned}
$$

where $\varrho_{f}=\int_{\mathbb{R}^{3}} f d \xi$ and $m_{f}=\int_{\mathbb{R}^{3}} \xi f d \xi$.

Proof. 1. We first apply the previous lemma and Lemma 3.1, to deduce that

$$
\varrho_{f_{n}} \in_{b} L^{p}\left(\mathbb{T}^{3} \times(0, T)\right), \quad m_{f_{n}} \in{ }_{b} L^{q}\left(\mathbb{T}^{3} \times(0, T)\right),
$$


for any $p \in\left(1, \frac{5}{3}\right)$ and $q \in\left(1, \frac{5}{4}\right)$. Using this, we apply the Hölder inequality to find that

$$
\begin{aligned}
& \left|\int_{0}^{T} \int_{\mathbb{T}^{3}} \partial_{t} u_{n} \cdot v d x d t\right| \leq\left\|u_{n}\right\|_{L^{3}\left(\mathbb{T}^{3} \times(0, T)\right)}\left\|\nabla u_{n}\right\|_{L^{2}\left(\mathbb{T}^{3} \times(0, T)\right)}\|v\|_{L^{6}\left(\mathbb{T}^{3} \times(0, T)\right)} \\
& +\mu\left\|\nabla u_{n}\right\|_{L^{2}\left(\mathbb{T}^{3} \times(0, T)\right)}\|\nabla v\|_{L^{2}\left(\mathbb{T}^{3} \times(0, T)\right)} \\
& +\left\|p_{n}\right\|_{L^{2}\left(\mathbb{T}^{3} \times(0, T)\right)}\|\nabla \cdot v\|_{L^{2}\left(\mathbb{T}^{3} \times(0, T)\right)} \\
& +\left\|m_{f_{n}}\right\|_{L^{\infty}\left(0, T ; L^{q}\left(\mathbb{T}^{3}\right)\right)}\|v\|_{L^{1}\left(0, T ; L^{\frac{q}{q-1}}\left(\mathbb{T}^{3}\right)\right)} \\
& +\left\|\varrho_{f_{n}} u_{n}\right\|_{L^{2}\left(0, T ; L^{q}\left(\mathbb{T}^{3}\right)\right)}\|v\|_{L^{2}\left(0, T ; L^{\frac{q}{q-1}}\left(\mathbb{T}^{3}\right)\right)},
\end{aligned}
$$

for $q<\frac{5}{4}$ and all $v \in\left[\mathcal{C}_{0}^{\infty}\left(\mathbb{T}^{3} \times(0, T)\right)\right]^{3}$. To bound the last norm in the right-hand side, we shall need the calculation

$$
\begin{aligned}
\left\|\varrho_{f_{n}} u_{n}\right\|_{L^{2}\left(0, T ; L^{q}\left(\mathbb{T}^{3}\right)\right)} & \leq\left\|\varrho_{n}\right\|_{L^{\infty}\left(0, T ; L^{5-q}\left(\mathbb{T}^{3}\right)\right)}\left\|u_{n}\right\|_{L^{2}\left(0, T ; L^{5}\left(\mathbb{T}^{3}\right)\right)} \\
& \leq C\left\|\varrho_{f_{n}}\right\|_{L^{\infty}\left(0, T ; L^{\frac{5 q}{5-q}}\left(\mathbb{T}^{3}\right)\right)}\left\|u_{n}\right\|_{L^{2}\left(0, T ; W^{1,2}\left(\mathbb{T}^{3}\right)\right)} .
\end{aligned}
$$

We notice that $\frac{5 q}{5-q}<\frac{5}{3}$ since $q<\frac{5}{4}$. By applying (4.18) in (4.17), using (4.16) and (4.11), and using $q=\frac{6}{5}$, we deduce that

$$
\partial_{t} u_{n} \in_{b} L^{\frac{6}{5}}\left(0, T ; W^{-1,2}\left(\mathbb{T}^{3}\right)\right) .
$$

Since in addition $u_{n} \in_{b} L^{2}\left(0, T ; W^{1,2}\left(\mathbb{T}^{3}\right)\right)$, we can apply the Aubin-Lions lemma to conclude

$$
u_{n} \rightarrow u \quad \text { a.e. and in } L^{2}\left(0, T ; L^{2}\left(\mathbb{T}^{3}\right)\right) \text {, as } n \rightarrow \infty .
$$

2. To conclude compactness of $\varrho_{f_{n}}$, we write the first equation in (4.1) in the form

$$
\partial_{t} f_{n}+\xi \cdot \nabla f_{n}=\nabla_{\xi} \cdot G_{n}+\sigma \Delta_{\xi} f_{n},
$$

where $G_{n}=f_{n}\left(\chi_{n}\left(u_{n}\right)+\chi_{n}\left(u_{f_{n}}^{\varepsilon}\right)-2 \xi\right)$. By direct calculation, using the uniform bounds (4.10), (4.11), and (4.18), we deduce

$$
\begin{aligned}
& \left\|G_{n}\right\|_{L^{q}\left(\mathbb{T}^{3} \times \mathbb{R}^{3} \times(0, T)\right)} \\
& \quad \leq\left\|f_{n} u_{n}\right\|_{L^{q}\left(\mathbb{T}^{3} \times \mathbb{R}^{3} \times(0, T)\right)}+\left\|f_{n} u_{f_{n}}\right\|_{L^{q}\left(\mathbb{T}^{3} \times \mathbb{R}^{3} \times(0, T)\right)}+2\left\|f_{n}|\xi|\right\|_{L^{q}\left(\mathbb{T}^{3} \times \mathbb{R}^{3} \times(0, T)\right)} \\
& \quad \leq C\left(\left\|u_{n}\right\|_{L^{q}\left(0, T ; L^{5}\left(\mathbb{T}^{3}\right)\right)}+\left\|m_{f_{n}}\right\|_{L^{q}\left(\mathbb{T}^{3} \times(0, T)\right)}+\left\|\sqrt{f_{n}|\xi|}\right\|_{L^{2}\left(\mathbb{T}^{3} \times \mathbb{R}^{3} \times(0, T)\right)}\right) \\
& \quad \leq C,
\end{aligned}
$$

where we used

$$
\begin{aligned}
\left\|f_{u} u_{n}\right\|_{L^{q}\left(\mathbb{T}^{3} \times \mathbb{R}^{3}\right)} & \leq\left\|f_{n}\right\|_{L^{\infty}\left(\mathbb{T}^{3} \times \mathbb{R}^{3}\right)}^{\frac{q-1}{q}}\left\|\rho_{f_{n}}^{\frac{1}{q}}\right\|_{L^{\frac{5 q}{5-q}\left(\mathbb{T}^{3}\right)}}\left\|u_{n}\right\|_{L^{5}\left(\mathbb{T}^{3}\right)} \\
& \left.\leq\left\|f_{n}\right\|_{L^{\infty}\left(\mathbb{T}^{3} \times \mathbb{R}^{3}\right)}^{\frac{q-1}{q}}\left\|\rho_{f_{n}}\right\|_{L^{\frac{1}{5-q}}}^{\frac{5}{q}} \| \mathbb{T}_{n}\right)
\end{aligned}
$$

and here $\left\|\rho_{f_{n}}\right\|_{L^{\frac{1}{5-q}}\left(\mathbb{T}^{3}\right)}^{\frac{1}{q}}$ is uniformly bounded in $n$ since $\frac{5}{5-q}<\frac{5}{3}$. Hence we can conclude that

$$
G_{n} \in L_{b} L^{q}\left(\mathbb{T}^{3} \times \mathbb{R}^{3} \times(0, T)\right), \quad \forall q \in\left(1, \frac{5}{4}\right) .
$$

The velocity averaging Lemma 3.2 is then applicable and yields

$$
\int_{\mathbb{R}^{3}} f_{n} \psi(\xi) d \xi \rightarrow \int_{\mathbb{R}^{3}} f \psi(\xi) d \xi \quad \text { in } L^{q}\left(\mathbb{T}^{3} \times(0, T)\right),
$$


for any $\psi(\xi)$ such that $|\psi(\xi)| \leq c|\xi|$ as $|\xi| \rightarrow \infty$, and any $q \in\left(1, \frac{5}{4}\right)$. If we set $\psi(\xi) \equiv 1$ and $\psi(\xi) \equiv \xi$ in (4.21), we obtain

$$
\varrho_{f_{n}} \rightarrow \varrho_{f} \quad \text { and } \quad m_{f_{n}} \rightarrow m_{f} \quad \text { in } L^{q}\left(\mathbb{T}^{3} \times(0, T)\right) .
$$

The proof is now complete.

In the next lemma, we establish convergence of solutions to (4.1) as $R \rightarrow \infty$. Specifically, we will send $n \rightarrow \infty$ in

$$
\begin{aligned}
& \partial_{t} f_{n}+\xi \cdot \nabla f_{n}+\nabla_{\xi} \cdot\left[f_{n}\left(\chi_{n}\left(u_{n}\right)-\xi\right)\right]=-\nabla_{\xi} \cdot\left[f_{n}\left(\chi_{n}\left(u_{f_{n}}^{\varepsilon}\right)-\xi\right)\right]+\sigma \Delta_{\xi} f_{n}, \\
& \partial_{t} u_{n}+\left(\theta_{\varepsilon} \star u_{n}\right) \cdot \nabla u_{n}+\nabla p_{n}=\mu \Delta u_{n}+\left(m_{f_{n}}-\varrho_{f_{n}} u_{n}\right) \mathbf{1}_{n}\left(u_{n}\right), \\
& \nabla \cdot u_{n}=0 .
\end{aligned}
$$

Lemma 4.5. Under the conditions of the previous lemma, $(f, u)$ is a weak solution of

$$
\begin{aligned}
& \partial_{t} f+\xi \cdot \nabla f+\nabla_{\xi} \cdot[f(u-\xi)]=-\nabla_{\xi} \cdot\left[f\left(u_{f}^{\varepsilon}-\xi\right)\right]+\sigma \Delta_{\xi} f, \\
& \partial_{t} u+\left(\theta_{\varepsilon} \star u\right) \cdot \nabla u+\nabla p=\mu \Delta u+\left(m_{f}-\varrho_{f} u\right), \\
& \nabla \cdot u=0,
\end{aligned}
$$

in the sense of Definition 2.1, where $u_{f}^{\varepsilon}$ is defined in (4.2). Moreover, $(f, u)$ satisfies

$$
\begin{aligned}
& \|f\|_{L^{\infty}\left(0, T ; L^{p}\left(\mathbb{T}^{3} \times \mathbb{R}^{3}\right)\right)}+\left\|\nabla_{\xi} f^{\frac{p}{2}}\right\|_{L^{2}\left(0, T ; L^{2}\left(\mathbb{T}^{3} \times \mathbb{R}^{3}\right)\right)}^{\frac{2}{p}} \leq C(p, T)\left\|f_{0}\right\|_{L^{p}\left(\mathbb{T}^{3} \times \mathbb{R}^{3}\right)}, \\
& \sup _{t \in(0, T)} \mathbb{E}(t)+\mu \int_{0}^{T}\|\nabla u\|_{L^{2}\left(\mathbb{T}^{3}\right)}^{2} d t+\int_{\mathbb{T}^{3} \times \mathbb{R}^{3}} f|u-\xi|^{2} d \xi d x \\
& \quad \leq \mathbb{E}(0)+3 \sigma M_{0}\left(f_{0}\right) T .
\end{aligned}
$$

Proof. The only problematic terms when passing to the limit in (4.22), are

$$
f_{n} \chi_{n}\left(u_{n}\right), \quad f_{n} \chi_{n}\left(u_{f_{n}}^{\varepsilon}\right), \quad \varrho_{f_{n}} u_{n} \mathbf{1}_{n}\left(u_{n}\right) .
$$

1. Let us begin with the latter. From (4.18), we have that

$$
\left\|\varrho_{f_{n}} u_{n}\right\|_{L^{2}\left(0, T ; L^{q}\left(\mathbb{T}^{3}\right)\right)} \leq C, \quad q<\frac{5}{4} .
$$

As a consequence, we can apply weak compactness to (4.27), (4.11) and use the strong convergences of $\rho_{f_{n}}$ and $u_{n}$ in (4.15) to deduce

$$
\varrho_{f_{n}} u_{n} \rightarrow \varrho_{f} u \quad \text { as } n \rightarrow \infty \text { in } L^{2}\left(0, T ; L^{q}\left(\mathbb{T}^{3}\right)\right), q<\frac{5}{4} .
$$

Now, by adding and subtracting, we see that

$$
\varrho_{f_{n}} u_{n} \mathbf{1}_{n}\left(u_{n}\right)=\varrho_{f_{n}} u_{n}-\varrho_{f_{n}} u_{n}\left(1-\mathbf{1}_{n}\left(u_{n}\right)\right),
$$

where the last term converges to zero as

$$
\begin{aligned}
& \left\|\varrho_{f_{n}} u_{n}\left(1-\mathbf{1}_{n}\left(u_{n}\right)\right)\right\|_{L^{1}\left(\mathbb{T}^{3} \times(0, T)\right)} \\
& \quad \leq C\left\|\varrho_{f_{n}} u_{n}\right\|_{L^{2}\left(0, T: L^{\frac{6}{5}}\left(\mathbb{T}^{3}\right)\right)}\left\|1-\mathbf{1}_{n}\left(u_{n}\right)\right\|_{L^{2}\left(0, T ; L^{6}\left(\mathbb{T}^{3}\right)\right)} \\
& \quad \leq \frac{C}{n}\left\|u_{n}\right\|_{L^{2}\left(0, T ; L^{6}\left(\mathbb{T}^{3}\right)\right)} \leq \frac{C}{n}\left\|u_{n}\right\|_{L^{2}\left(0, T ; H^{1}\left(\mathbb{T}^{3}\right)\right)} \stackrel{n \rightarrow \infty}{\longrightarrow} 0,
\end{aligned}
$$

where we have used $q=\frac{6}{5}<\frac{5}{4}$ in (4.18) and the estimates of uniform bounds for the approximations (4.11). Hence, we can send $n \rightarrow \infty$ in (4.29) to conclude

$$
\varrho_{f_{n}} u_{n} \mathbf{1}_{n}\left(u_{n}\right) \rightarrow \varrho_{f} u \quad \text { as } n \rightarrow \infty \text { in } L^{2}\left(0, T ; L^{q}\left(\mathbb{T}^{3}\right)\right), q<\frac{5}{4} .
$$


2. Let us now consider the first term in (4.26). For this purpose, we use (4.11) and (4.19) to find

$$
\begin{aligned}
& \left\|f_{u} u_{n}\right\|_{L^{2}\left(0, T ; L^{q}\left(\mathbb{T}^{3} \times \mathbb{R}^{3}\right)\right)} \\
& \quad \leq\left\|f_{n}\right\|_{L^{\infty}\left(\mathbb{T}^{3} \times \mathbb{R}^{3} \times(0, T)\right)}^{\frac{q-1}{q}}\left\|\rho_{f_{n}}\right\|^{\frac{1}{q}} L^{\infty}\left(0, T ; L^{5-q}\left(\mathbb{T}^{3}\right)\right) \\
& \quad \leq C\left\|u_{n}\right\|_{L^{2}\left(0, T ; H^{1}\left(\mathbb{T}^{3}\right)\right)} \leq C .
\end{aligned}
$$

Then we use the similar argument to (4.28) to obtain

$$
f_{n} u_{n} \rightarrow f u \quad \text { in } L^{2}\left(0, T ; L^{q}\left(\mathbb{T}^{3} \times \mathbb{R}^{3}\right)\right) .
$$

Next, by adding and subtracting, we write

$$
f_{n} \chi_{n}\left(u_{n}\right)=f_{n} u_{n}+f_{n}\left(\chi_{n}\left(u_{n}\right)-u_{n}\right) \text {, }
$$

where the last term converges to zero as

$$
\begin{aligned}
& \left\|f_{n}\left(\chi_{n}\left(u_{n}\right)-u_{n}\right)\right\|_{L^{1}\left((0, T) \times \mathbb{T}^{3} \times \mathbb{R}^{3}\right)} \\
& \quad=\left\|\varrho_{f_{n}} u_{n}\left(1-\mathbf{1}_{n}\left(u_{n}\right)\right)\right\|_{L^{1}\left((0, T) \times \mathbb{T}^{3}\right)} \\
& \quad \leq\left\|\varrho_{f_{n}}\right\|_{L^{\infty}\left(0, T ; L^{\frac{3}{2}}\left(\mathbb{T}^{3}\right)\right)}\left\|u_{n}\right\|_{L^{2}\left(0, T ; L^{6}\left(\mathbb{T}^{3}\right)\right)}\left\|1-\mathbf{1}_{n}\left(u_{n}\right)\right\|_{L^{2}\left(0, T ; L^{6}\left(\mathbb{T}^{3}\right)\right)} \\
& \quad \leq \frac{1}{n}\left\|\varrho_{f_{n}}\right\|_{L^{\infty}\left(0, T ; L^{\frac{3}{2}}\left(\mathbb{T}^{3}\right)\right)}\left\|u_{n}\right\|_{L^{2}\left(0, T ; L^{6}\left(\mathbb{T}^{3}\right)\right)}^{2} \stackrel{n \rightarrow \infty}{\longrightarrow} 0 .
\end{aligned}
$$

This, together with (4.31), in (4.32) yields

$$
f_{n} \chi_{n}\left(u_{n}\right) \rightarrow f u \quad \text { in } L^{2}\left(0, T ; L^{q}\left(\mathbb{T}^{3} \times \mathbb{R}^{3}\right)\right) .
$$

3. Finally, we consider the second term in (4.26). First, we calculate

$$
\begin{aligned}
\left\|f_{n} u_{f_{n}}^{\varepsilon}\right\|_{L^{\infty}\left(0, T ; L^{q}\left(\mathbb{T}^{3} \times \mathbb{R}^{3}\right)\right)} & \leq\left\|f_{n}\right\|_{L^{\infty}\left(0, T ; L^{\infty}\left(\mathbb{T}^{3} \times \mathbb{R}^{3}\right)\right)}\left\|u_{f_{n}}^{\varepsilon}\right\|_{L^{\infty}\left(0, T ; L^{q}\left(\mathbb{T}^{3}\right)\right)} \\
& \leq \frac{C}{\varepsilon}\left\|m_{f_{n}}\right\|_{L^{\infty}\left(0, T ; L^{q}\left(\mathbb{T}^{3}\right)\right)} \leq \frac{C}{\varepsilon}
\end{aligned}
$$

where the last inequality is (4.10), (4.16), and $q<\frac{5}{4}$. We also notice that the convergence estimates of $\rho_{f_{n}}$ and $m_{f_{n}}$ in (4.15) and $\frac{1}{\varepsilon+\rho_{f_{n}}} \leq \frac{1}{\varepsilon}$ yield

$$
u_{f_{n}}^{\varepsilon}=\frac{m_{f_{n}}}{\varepsilon+\varrho_{f_{n}}} \stackrel{n \rightarrow \infty}{\longrightarrow} \frac{m_{f}}{\varepsilon+\varrho_{f}} \quad \text { in } L^{q}\left(\mathbb{T}^{3} \times(0, T)\right), q<\frac{5}{4},
$$

for each fixed $\varepsilon>0$, due to a simple application of Vitali's convergence theorem. In particular, we again use the similar strategy to (4.28) to have that

$$
f_{n} u_{f_{n}}^{\varepsilon} \rightarrow f u_{f}^{\varepsilon} \quad \text { as } n \rightarrow \infty \text { in } L^{q}\left(\mathbb{T}^{3} \times(0, T)\right) .
$$

By adding and subtracting,

$$
f_{n} \chi_{n}\left(u_{f_{n}}^{\varepsilon}\right)=f_{n} u_{f_{n}}^{\varepsilon}+f_{n}\left(\chi_{n}\left(u_{f_{n}}^{\varepsilon}\right)-u_{f_{n}}^{\varepsilon}\right)
$$

where the last term satisfies

$$
\begin{aligned}
& \left\|f_{n}\left(\chi_{n}\left(u_{f_{n}}^{\varepsilon}\right)-u_{f_{n}}^{\varepsilon}\right)\right\|_{L^{1}\left(\mathbb{T}^{3} \times \mathbb{R}^{3} \times(0, T)\right)} \\
& \quad=\left\|\varrho_{f_{n}} u_{f_{n}}^{\varepsilon}\left(1-\mathbf{1}_{n}\left(u_{f_{n}}^{\varepsilon}\right)\right)\right\|_{L^{1}\left(\mathbb{T}^{3} \times(0, T)\right)} \leq \frac{1}{n} \int_{0}^{T} \int_{\mathbb{T}^{3}} \varrho_{f_{n}}\left|u_{f_{n}}^{\varepsilon}\right|^{2} d x d t \stackrel{n \rightarrow \infty}{\longrightarrow} 0,
\end{aligned}
$$

since $\int_{\mathbb{T}^{3}} \varrho_{f_{n}}\left|u_{f_{n}}^{\varepsilon}\right|^{2} d x \leq \int_{\mathbb{T}^{3} \times \mathbb{R}^{3}} f_{n}|\xi|^{2} d \xi d x$, which is bounded by (4.11). By combining (4.34), (4.35), and (4.36), we conclude

$$
f_{n} \chi_{n}\left(u_{f_{n}}^{\varepsilon}\right) \rightarrow f u_{f}^{\varepsilon} \quad \text { as } n \rightarrow \infty \text { in } L^{q}\left(\mathbb{T}^{3} \times(0, T)\right)
$$


4. Equipped with (4.30), (4.33), and (4.37), there are no problems with passing to the limit in (4.22) to conclude that $(u, f)$ is a weak solution to (4.23). The bounds (4.24) and (4.25) can be proved as in Propositions 3.1 and 3.2.

\subsection{The $\varepsilon \rightarrow 0$ limit and proof of Theorem 2.1}

We will now send $\varepsilon \rightarrow 0$ in (4.23) and thereby conclude the proof of Theorem 2.1. The largest challenge is presented by possible vacuum regions of $\varrho_{f}$ rendering passing to the limit in $u_{f}$ non-trivial. To this end, we shall need the following lemma:

Lemma 4.6. Let $\left\{\left(f_{\varepsilon}, u_{\varepsilon}\right)\right\}_{\varepsilon>0}$ be a sequence of weak solutions to (4.23). As $\varepsilon \rightarrow 0$,

$$
\begin{aligned}
& f_{\varepsilon} \rightarrow f \quad \text { in } \mathcal{C}\left([0, T] ; L^{p}\left(\mathbb{T}^{3} \times \mathbb{R}^{3}\right)\right), p \in(1, \infty), \\
& \varrho_{f_{\varepsilon}} \rightarrow \varrho_{f} \quad \text { a.e. and in } L^{p}\left(\mathbb{T}^{3} \times(0, T)\right), p \in\left(1, \frac{5}{4}\right), \\
& m_{f_{\varepsilon}} \rightarrow m_{f} \quad \text { a.e. and in } L^{q}\left(\mathbb{T}^{3} \times(0, T)\right), q \in\left(1, \frac{5}{4}\right), \\
& u_{\varepsilon} \rightarrow u \quad \text { a.e. and in } L^{2}\left(\mathbb{T}^{3} \times(0, T)\right),
\end{aligned}
$$

where $\varrho_{f}=\int_{\mathbb{R}^{3}} f d \xi, m_{f}=\int_{\mathbb{R}^{3}} \xi f d \xi$, and where the convergence may take place along a subsequence.

Proof. Since (4.16), (4.18), and (4.20) hold independently of $\varepsilon$, the proof follows by the exact same arguments as the proof of Lemma 4.4.

Theorem 2.1 follows as a consequence of the following lemma.

Lemma 4.7. Under the conditions of the previous lemma, $(f, u)$ is a weak solution of $(1.1)$ in the sense of Definition 2.1, where

$$
u_{f}= \begin{cases}\frac{\int_{\mathbb{R}^{3}} f \xi d \xi}{\int_{\mathbb{R}^{3}} f d \xi}, & \varrho_{f} \neq 0, \\ 0, & \varrho_{f}=0 .\end{cases}
$$

Proof. From (4.38), we easily conclude that

$$
\begin{aligned}
& f_{\varepsilon}\left(u_{\varepsilon}-\xi\right) \rightarrow f(u-\xi) \quad \text { in } L^{2}\left(0, T ; L^{q}\left(\mathbb{T}^{3} \times \mathbb{R}^{3}\right)\right), q<\frac{5}{4}, \\
& m_{f_{\varepsilon}}-\varrho_{f_{\varepsilon}} u_{\varepsilon} \rightarrow m_{f}-\varrho_{f} u \quad \text { in } L^{2}\left(0, T ; L^{q}\left(\mathbb{T}^{3}\right)\right), q<\frac{5}{4}, \\
& \left(\theta_{\varepsilon} \star u_{\varepsilon}\right) \cdot \nabla u_{\varepsilon} \rightarrow u \cdot \nabla u \quad \text { in } L^{\frac{6}{5}}\left(\mathbb{T}^{3} \times(0, T)\right) .
\end{aligned}
$$

Hence, in order to pass to the limit in (4.23), it remains to prove that

$$
f_{\varepsilon} u_{f_{\varepsilon}}^{\varepsilon} \rightarrow f u_{f} \quad \text { as } \varepsilon \rightarrow 0 \text { in the sense of distribution. }
$$

For this purpose, let $\lambda>0$ be a small parameter and define

$$
A_{\lambda}=\left\{(x, t): \varrho_{f}(x, t)>\lambda\right\} .
$$

Since $\varrho_{f_{\varepsilon}} \rightarrow \varrho_{f}$ a.e., Egoroff's theorem yields, for any $\eta>0$, the existence of a set $B_{\lambda, \eta}$ with $\left|A_{\lambda} \backslash B_{\lambda, \eta}\right|<\eta$ and where $\varrho_{f_{\varepsilon}} \rightarrow \varrho_{f}$ uniformly on $B_{\lambda, \eta}$. In particular, for a sufficiently small $\bar{\varepsilon}$,

$$
\varrho_{f_{\varepsilon}}>\lambda-\frac{\eta}{2}, \quad \forall \varepsilon<\bar{\varepsilon}, \quad(x, t) \in B_{\lambda, \eta} .
$$


By virtue of (4.38), we have that

$$
u_{f_{\varepsilon}}^{\varepsilon}=\frac{m_{f_{\varepsilon}}}{\varepsilon+\varrho_{f_{\varepsilon}}} \rightarrow \frac{m_{f}}{\varrho_{f}} \quad \text { in } L^{q}\left(B_{\lambda, \eta}\right), q<\frac{5}{4} .
$$

In particular, since $f_{\varepsilon}$ converges weakly, we can conclude that

$$
f_{\varepsilon} u_{f_{\varepsilon}}^{\varepsilon} \rightarrow f u_{f} \quad \text { in } L^{q}\left(B_{\lambda, \eta} \times \mathbb{R}^{3}\right) \text { as } \varepsilon \rightarrow 0 .
$$

We then write

$$
f_{\varepsilon} u_{f_{\varepsilon}}^{\varepsilon} \mathbf{1}_{A_{\lambda}}=f_{\varepsilon} u_{f_{\varepsilon}}^{\varepsilon} \mathbf{1}_{B_{\eta}}+f_{\varepsilon} u_{f_{\varepsilon}}^{\varepsilon} \mathbf{1}_{A_{\lambda} \backslash B_{\eta}},
$$

where we see that the last term is small due to the following bound

$$
\left\|f_{\varepsilon} u_{f_{\varepsilon}}^{\varepsilon} \mathbf{1}_{A_{\lambda} \backslash B_{\eta}}\right\|_{L^{1}\left(\mathbb{T}^{3} \times \mathbb{R}^{3} \times(0, T)\right)} \leq \eta^{\frac{1}{q^{\prime}}}\left\|m_{f_{\varepsilon}}\right\|_{L^{q}\left(\mathbb{T}^{3} \times(0, T)\right)}=\mathcal{O}\left(\eta^{\frac{1}{q^{\prime}}}\right) .
$$

Since we can choose $\eta$ arbitrarily small, we must have that

$$
f_{\varepsilon} u_{f_{\varepsilon}}^{\varepsilon} \rightarrow f u_{f} \quad \text { in } L^{q}\left(A_{\lambda} \times \mathbb{R}^{3}\right) \text { as } \varepsilon \rightarrow 0 .
$$

For the estimate on the set $\left(\mathbb{T}^{3} \times(0, T)\right) \backslash A_{\lambda}$, we let $\eta$ be a small parameter and make another application of Egoroff's theorem to obtain a set $C_{\lambda, \eta}$ such that

$$
\left|\left(\left(\mathbb{T}^{3} \times(0, T)\right) \backslash A_{\lambda}\right) \backslash C_{\lambda, \eta}\right|<\eta, \quad \varrho_{f_{\varepsilon}}<\lambda+\frac{\eta}{2}, \quad \forall \varepsilon<\bar{\varepsilon}
$$

On $C_{\lambda, \eta}$, the product $\varrho_{f_{n}} u_{f_{n}}$ is controlled by $\lambda+\frac{\eta}{2}$ as

$$
\begin{aligned}
\left\|f_{\varepsilon} u_{f_{\varepsilon}}^{\varepsilon}\right\|_{L^{1}\left(C_{\lambda, \eta} \times \mathbb{R}^{3}\right)} & \leq\left(\int_{C_{\lambda, \eta}} \varrho_{f_{\varepsilon}} d x d t\right)^{\frac{1}{2}}\left(\int_{0}^{T} \int_{\mathbb{T}^{3}} \varrho_{f_{\varepsilon}}\left|u_{f_{\varepsilon}}^{\varepsilon}\right|^{2} d x d t\right)^{\frac{1}{2}} \\
& \leq\left(\lambda+\frac{\eta}{2}\right)^{\frac{1}{2}} C,
\end{aligned}
$$

where we have used $\int_{\mathbb{T}^{3}} \varrho_{f_{\varepsilon}}\left|u_{f_{\varepsilon}}^{\varepsilon}\right|^{2} d x \leq \int_{\mathbb{T}^{3} \times \mathbb{R}^{3}} f_{\varepsilon}|\xi|^{2} d \xi d x$ which is bounded by (4.11), and the fact that $\left|\mathbb{T}^{3} \times(0, T)\right|$ is finite to conclude the last inequality. As in (4.39), we also see that

$$
\left\|f_{\varepsilon} u_{f_{\varepsilon}}^{\varepsilon}\right\|_{L^{1}\left(\left(\left(\left(\mathbb{T}^{3} \times(0, T)\right) \backslash A_{\lambda}\right) \backslash C_{\lambda, \eta}\right) \times \mathbb{R}^{3}\right)} \leq \mathcal{O}\left(\eta^{\frac{1}{q^{\prime}}}\right) .
$$

Since $\eta$ can be chosen arbitrarily small, we deduce

$$
\left\|f_{\varepsilon} u_{f_{\varepsilon}}^{\varepsilon}\right\|_{L^{1}\left(\left(\left(\mathbb{T}^{3} \times(0, T)\right) \backslash A_{\lambda}\right) \times \mathbb{R}^{3}\right)} \leq \mathcal{O}\left(\lambda^{\frac{1}{2}}\right) .
$$

Hence by choosing sufficiently small $\lambda$ to conclude

$$
f_{\varepsilon} u_{f_{\varepsilon}}^{\varepsilon} \rightarrow f u_{f} \quad \text { in } L^{q}\left(\mathbb{T}^{3} \times \mathbb{R}^{3} \times(0, T)\right), q<\frac{5}{4} .
$$

This completes the proof.

\section{Hydrodynamic limit (Theorem 2.2)}

In this section, we will study the flocking-Navier-Stokes system (1.1) under the assumption of strong noise and local alignment. In this regime, we shall rigorously establish that the evolution can be accurately described by a coupled compressible Euler and incompressible Navier-Stokes system, and thereby prove Theorem 2.2. For the reader's convenience, we recall the equations under consideration: 


$$
\begin{aligned}
& \partial_{t} f^{\varepsilon}+\xi \cdot \nabla f^{\varepsilon}+\nabla_{\xi} \cdot\left[\left(u^{\varepsilon}-\xi\right) f^{\varepsilon}\right]=\frac{1}{\varepsilon} \nabla_{\xi} \cdot\left[\nabla_{\xi} f^{\varepsilon}-\left(u_{f^{\varepsilon}}-\xi\right) f^{\varepsilon}\right], \\
& \partial_{t} u^{\varepsilon}+u^{\varepsilon} \cdot \nabla u^{\varepsilon}+\nabla p^{\varepsilon}-\mu \Delta u^{\varepsilon}=-\int_{\mathbb{R}^{3}}\left(u^{\varepsilon}-\xi\right) f^{\varepsilon} d \xi \\
& \nabla \cdot u^{\varepsilon}=0
\end{aligned}
$$

subject to

$$
\left(f^{\varepsilon}(x, \xi, 0), u^{\varepsilon}(x, 0)\right)=\left(f_{0}(x, \xi), u_{0}(x)\right) .
$$

Our goal is to prove that solutions of this system can be well approximated by the Euler-Navier-Stokes system

$$
\begin{aligned}
& \partial_{t} u_{f}+\nabla \cdot\left(\varrho_{f} u_{f}\right)=0, \\
& \left(\varrho_{f} u_{f}\right)_{t}+\nabla \cdot\left(\varrho_{f} u_{f} \otimes u_{f}\right)+\nabla \varrho_{f}=\varrho_{f}\left(u-u_{f}\right), \\
& u_{t}+u \cdot \nabla u+\nabla p-\mu \Delta u=-\varrho_{f}\left(u-u_{d}\right), \\
& \nabla \cdot u=0,
\end{aligned}
$$

provided $\varepsilon$ is sufficiently small.

\subsection{Entropy of weak solutions}

We first show that the weak solutions obtained from Theorem 2.1 satisfies some entropy inequalities that are uniform in $\varepsilon$. For this, we set

$$
\begin{aligned}
\mathcal{F}\left(f^{\varepsilon}, u^{\varepsilon}\right) & :=\int_{\mathbb{T}^{3} \times \mathbb{R}^{3}} f^{\varepsilon}\left(\log f^{\varepsilon}+\frac{|\xi|^{2}}{2}\right) d x d \xi+\int_{\mathbb{T}^{3}} \frac{\left|u^{\varepsilon}\right|^{2}}{2} d x, \\
D_{1}\left(f^{\varepsilon}\right) & :=\int_{\mathbb{T}^{3} \times \mathbb{R}^{3}} \frac{1}{f^{\varepsilon}}\left|\nabla_{\xi} f^{\varepsilon}+\left(u_{f^{\varepsilon}}-\xi\right) f^{\varepsilon}\right|^{2} d x d \xi, \\
D_{2}\left(f^{\varepsilon}, u^{\varepsilon}\right) & :=\int_{\mathbb{T}^{3} \times \mathbb{R}^{3}}\left|u^{\varepsilon}-\xi\right|^{2} f^{\varepsilon} d x d \xi+\mu \int_{\mathbb{T}^{3}}\left|\nabla u^{\varepsilon}\right|^{2} d x .
\end{aligned}
$$

Then it follows from Proposition 3.1 that

$$
\frac{d}{d t} \mathcal{F}\left(f^{\varepsilon}, u^{\varepsilon}\right)+\frac{1}{\varepsilon} D_{1}\left(f^{\varepsilon}\right)+D_{2}\left(f^{\varepsilon}, u^{\varepsilon}\right)=3 M_{0}\left(f_{0}\right)
$$

and this yields

$$
\mathcal{F}\left(f^{\varepsilon}, u^{\varepsilon}\right)-\mathcal{F}\left(f_{0}, u_{0}\right)+\frac{1}{\varepsilon} \int_{0}^{t} D_{1}\left(f^{\varepsilon}\right) d s+\int_{0}^{t} D_{2}\left(f^{\varepsilon}, u^{\varepsilon}\right) d s=3 M_{0}\left(f_{0}\right) t .
$$

On the other hand, we notice that

$$
\int_{\mathbb{T}^{3} \times \mathbb{R}^{3}} f^{\varepsilon}\left|\log f^{\varepsilon}\right| d x d \xi \leq \int_{\mathbb{T}^{3} \times \mathbb{R}^{3}} f^{\varepsilon} \log f^{\varepsilon} d x d \xi+\frac{1}{4} \int_{\mathbb{T}^{3} \times \mathbb{R}^{3}}\left(1+|\xi|^{2}\right) f^{\varepsilon} d x d \xi+C .
$$

This implies that

$$
\begin{aligned}
& \int_{\mathbb{T}^{3} \times \mathbb{R}^{3}} f^{\varepsilon}\left(1+\left|\log f^{\varepsilon}\right|+\frac{1}{4}|\xi|^{2}\right) d x d \xi+\int_{\mathbb{T}^{3}} \frac{\left|u^{\varepsilon}\right|^{2}}{2} d x \\
& +\int_{0}^{T} D_{2}\left(f^{\varepsilon}, u^{\varepsilon}\right) d t+\frac{1}{\varepsilon} \int_{0}^{T} D_{1}\left(f^{\varepsilon}\right) d t \leq \mathcal{F}\left(f_{0}, u_{0}\right)+C(T) .
\end{aligned}
$$


By expanding the square, one can check after some tedious computations that

$$
\begin{aligned}
& \frac{1}{2} \int_{\mathbb{T}^{6} \times \mathbb{R}^{6}} f^{\varepsilon}(x, \xi) f^{\varepsilon}\left(y, \xi_{*}\right)\left|\xi-\xi_{*}\right|^{2} d x d \xi d y d \xi_{*}+\int_{\mathbb{T}^{3}} \rho_{f^{\varepsilon}}\left|u^{\varepsilon}-u_{f^{\varepsilon}}\right|^{2} d x \\
& \quad=\int_{\mathbb{T}^{3} \times \mathbb{R}^{3}}\left|u^{\varepsilon}-\xi\right|^{2} f^{\varepsilon} d x d \xi+\frac{1}{2} \int_{\mathbb{T}^{3} \times \mathbb{T}^{3}} \rho_{f^{\varepsilon}}(x) \rho_{f^{\varepsilon}}(y)\left|u_{f^{\varepsilon}}(x)-u_{f^{\varepsilon}}(y)\right|^{2} d x d y .
\end{aligned}
$$

Now we use the similar estimates in [22, Lemma B.3] to get

$$
\begin{aligned}
& \frac{1}{2} \int_{\mathbb{T}^{3} \times \mathbb{T}^{3}} \rho_{f^{\varepsilon}}(x) \rho_{f^{\varepsilon}}(y)\left|u_{f^{\varepsilon}}(x)-u_{f^{\varepsilon}}(y)\right|^{2} d x d y \\
& \quad \leq-3\left(M_{0}\left(f_{0}\right)\right)^{2}+C(T) \varepsilon+\frac{1}{2 \varepsilon} D_{1}\left(f^{\varepsilon}\right)+\int_{\mathbb{T}^{6} \times \mathbb{R}^{6}} f^{\varepsilon}(x, \xi) f^{\varepsilon}\left(y, \xi_{*}\right) \frac{\left|\xi-\xi_{*}\right|^{2}}{2} d x d \xi d y d \xi_{*} .
\end{aligned}
$$

Combining (5.4), (5.6), and (5.7), we obtain

$$
\begin{aligned}
& \mathcal{F}\left(f^{\varepsilon}, u^{\varepsilon}\right)+\frac{1}{2 \varepsilon} \int_{0}^{t} D_{1}\left(f^{\varepsilon}\right) d s+\int_{0}^{t} \int_{\mathbb{T}^{3}} \rho_{f^{\varepsilon}}\left|u^{\varepsilon}-u_{f^{\varepsilon}}\right|^{2} d x d s+\mu \int_{0}^{t} \int_{\mathbb{T}^{3}}\left|\nabla u^{\varepsilon}\right|^{2} d x d s \\
& \quad \leq \mathcal{F}\left(f_{0}, u_{0}\right)+C(T) \varepsilon,
\end{aligned}
$$

where we used the fact that $f_{0}$ has a unit mass, i.e., $M_{0}\left(f_{0}\right)=1$. In light of the above arguments, we conclude the following proposition.

Proposition 5.1. Suppose the initial data $\left(f_{0}, u_{0}\right)$ satisfies (2.1). Then for any $T>0$ and $\varepsilon>0$ there exists at least one weak solution $\left(f^{\mathcal{E}}, u^{\varepsilon}\right)$ to (5.1)-(5.2) on the time-interval $(0, T)$ satisfying (5.5) and (5.8).

We will prove Theorem 2.2 through a relative entropy argument. For this to be rigorous, we need a unique strong solution (at least for short time) to the system (2.3)-(2.4). We claim the following result.

Theorem 5.1. Let $s \geq 3$. Suppose the initial data $\left(\rho_{f_{0}}, u_{f_{0}}, u_{0}\right) \in H^{s}\left(\mathbb{T}^{3}\right)$ and $\rho_{f_{0}}>0$. Then there exists a positive constant $T^{*}>0$ such that the Cauchy problem (2.3)-(2.4) has a unique solution $\left(\rho_{f}, u_{f}, u\right)$ satisfying

$$
\begin{aligned}
\left(\rho_{f}, u_{f}\right) & \in \mathcal{C}\left(\left[0, T^{*}\right] ; H^{s}\left(\mathbb{T}^{3}\right)\right) \cap \mathcal{C}^{1}\left(\left[0, T^{*}\right] ; H^{s-1}\left(\mathbb{T}^{3}\right)\right), \\
u & \in \mathcal{C}\left(\left[0, T^{*}\right] ; H^{s}\left(\mathbb{T}^{3}\right)\right) \cap L^{2}\left(0, T^{*} ; H^{s+1}\left(\mathbb{T}^{3}\right)\right) .
\end{aligned}
$$

Since local existence theories for this type of balance laws have been well developed, we omit this proof. We refer to [25] for the readers who are interested in it.

\subsection{Relative entropy}

We shall prove Theorem 2.2 using a relative entropy argument. For this purpose, it will be convenient to write the equation in a more abstract form using the variables

$$
U:=\left(\begin{array}{c}
\varrho_{f} \\
m_{f} \\
u
\end{array}\right), \quad A(U):=\left(\begin{array}{ccc}
m_{f} & 0 & 0 \\
\frac{m_{f} \otimes m_{f}}{\varrho_{f}} & \varrho_{f} & 0 \\
u \otimes u & 0 & 0
\end{array}\right),
$$

and

$$
F(U):=\left(\begin{array}{c}
0 \\
\varrho_{f} u-m_{f} \\
m_{f}-\varrho_{f} u-\nabla p+\mu \Delta u
\end{array}\right)
$$


where $m_{f}=\rho_{f} u_{f}$. The system can then be recast in the form

$$
U_{t}+\nabla \cdot A(U)=F(U),
$$

and the macroscopic entropy (energy) takes the form

$$
E(U):=\varrho_{f} \log \varrho_{f}+\frac{\left|m_{f}\right|^{2}}{2 \varrho_{f}}+\frac{|u|^{2}}{2} .
$$

Using the newly defined variables, we define the relative entropy functional as follows:

$$
\mathcal{H}(V \mid U):=E(V)-E(U)-d E(U)(V-U), \quad \text { and } \quad V:=\left(\begin{array}{c}
\varrho_{\bar{f}} \\
m_{\bar{f}} \\
\bar{u}
\end{array}\right) .
$$

Upon noticing that

$$
\begin{aligned}
-d E(U)(V-U) & =-\left(\begin{array}{c}
-\frac{m_{f}^{2}}{2 \rho_{f}^{2}}+\log \rho_{f}+1 \\
\frac{m_{f}}{\rho_{f}} \\
u
\end{array}\right)\left(\begin{array}{c}
\rho_{\bar{f}}-\rho_{f} \\
m_{\bar{f}}-m_{f} \\
\bar{u}-u
\end{array}\right) \\
& =\frac{\rho_{\bar{f}}}{2}\left|u_{f}\right|^{2}-\frac{\rho_{f}}{2}\left|u_{f}\right|^{2}-\left(\log \rho_{f}+1\right)\left(\rho_{\bar{f}}-\rho_{f}\right)+\rho_{f}\left|u_{f}\right|^{2}-\rho_{\bar{f}} u_{\bar{f}} \cdot u_{f}-(\bar{u}-u) \cdot u,
\end{aligned}
$$

we see that the relative entropy can alternatively be written

$$
\mathcal{H}(V \mid U)=\frac{\rho_{\bar{f}}}{2}\left|u_{f}-u_{\bar{f}}\right|^{2}+\frac{1}{2}|\bar{u}-u|^{2}+P\left(\rho_{\bar{f}}, \rho_{f}\right),
$$

where

$$
P\left(\rho_{\bar{f}}, \rho_{f}\right):=\rho_{\bar{f}} \log \rho_{\bar{f}}-\rho_{f} \log \rho_{f}+\left(\rho_{f}-\rho_{\bar{f}}\right)\left(1+\log \rho_{f}\right) \geq \frac{1}{2} \min \left\{\frac{1}{\rho_{\bar{f}}}, \frac{1}{\rho_{f}}\right\}\left(\rho_{\bar{f}}-\rho_{f}\right)^{2} .
$$

Hence, the relative entropy controls the $L^{2}$-difference provided one of the densities is without vacuum regions.

To proceed, we shall need to derive an evolution equation for the integrated relative entropy.

Lemma 5.1. The relative entropy $\mathcal{H}$ defined in (5.9) satisfies the following equality

$$
\begin{aligned}
& \frac{d}{d t} \int_{\mathbb{T}^{3}} \mathcal{H}(V \mid U) d x+\mu \int_{\mathbb{T}^{3}}|\nabla(u-\bar{u})|^{2} d x+\int_{\mathbb{T}^{3}} \rho_{\bar{f}}\left|\left(u_{\bar{f}}-\bar{u}\right)-\left(u_{f}-u\right)\right|^{2} d x \\
& =\int_{\mathbb{T}^{3}} \partial_{t} E(V) d x+\int_{\mathbb{T}^{3}} \rho_{\bar{f}}\left|\bar{u}-u_{\bar{f}}\right|^{2} d x+\mu \int_{\mathbb{T}^{3}}|\nabla \bar{u}|^{2} d x \\
& \quad-\int_{\mathbb{T}^{3}} \nabla(d E(U)): A(V \mid U) d x-\int_{\mathbb{T}^{3}} d E(U)\left[V_{t}+\nabla \cdot A(V)-F(V)\right] d x \\
& \quad-\int_{\mathbb{T}^{3}}\left(\rho_{f}-\rho_{\bar{f}}\right)(\bar{u}-u)\left(u-u_{f}\right) d x,
\end{aligned}
$$

where we have introduced the relative flux functional

$$
A(V \mid U):=A(V)-A(U)-d A(U)(V-U) .
$$

Proof. Although this lemma is essential for the proof of Theorem 2.2, it is rather lengthy and technical. Thus we postpone its proof in Appendix A for the smooth flow of reading. 


\subsection{Relative entropy bound}

The proof of Theorem 2.2 will follow as a consequence of the following proposition.

Proposition 5.2. Suppose all assumptions in Theorem 2.2. Set

$$
U:=\left(\begin{array}{c}
\rho_{f} \\
\rho_{f} u_{f} \\
u
\end{array}\right) \quad \text { and } \quad U^{\varepsilon}:=\left(\begin{array}{c}
\rho_{f^{\varepsilon}} \\
\rho_{f^{\varepsilon}} u_{f^{\varepsilon}} \\
u^{\varepsilon}
\end{array}\right),
$$

where $\left(\rho_{f}, u_{f}, u\right)$ and $\left(f^{\varepsilon}, u^{\varepsilon}\right)$ are a unique strong solution to the system (2.3)-(2.4) and weak solutions to the system (5.1)-(5.2), respectively. Then we have

$$
\begin{aligned}
& \int_{\mathbb{T}^{3}} \mathcal{H}\left(U^{\varepsilon} \mid U\right)(t) d x+\mu \int_{0}^{t} \int_{\mathbb{T}^{3}}\left|\nabla\left(u-u^{\varepsilon}\right)\right|^{2} d x d s+\frac{1}{2} \int_{0}^{t} \int_{\mathbb{T}^{3}} \rho_{f^{\varepsilon}}\left|\left(u_{f^{\varepsilon}}-u^{\varepsilon}\right)-\left(u_{f}-u\right)\right|^{2} d x d s \\
& \quad \leq C \sqrt{\varepsilon},
\end{aligned}
$$

for all $t \in\left[0, T^{*}\right]$.

Proof. From Lemma 5.1, we know that

$$
\begin{aligned}
& \int_{\mathbb{T}^{3}} \mathcal{H}\left(U^{\varepsilon} \mid U\right)(t) d x+\mu \int_{0}^{t} \int_{\mathbb{T}^{3}}\left|\nabla\left(u-u^{\varepsilon}\right)\right|^{2} d x d s+\int_{0}^{t} \int_{\mathbb{T}^{3}} \rho_{f^{\varepsilon}}\left|\left(u_{f^{\varepsilon}}-u^{\varepsilon}\right)-\left(u_{f}-u\right)\right|^{2} d x d s \\
& =\int_{0}^{t} \int_{\mathbb{T}^{3}} \partial_{t} E\left(U^{\varepsilon}\right)+\rho_{f^{\varepsilon}}\left|u^{\varepsilon}-u_{f^{\varepsilon}}\right|^{2}+\mu\left|\nabla u^{\varepsilon}\right|^{2} d x d s-\int_{0}^{t} \int_{\mathbb{T}^{3}} \nabla(d E(U)): A\left(U^{\varepsilon} \mid U\right) d x d s \\
& \quad-\int_{0}^{t} \int_{\mathbb{T}^{3}} d E(U)\left[U_{t}^{\varepsilon}+\nabla \cdot A\left(U^{\varepsilon}\right)-F\left(U^{\varepsilon}\right)\right] d x d s-\int_{0}^{t} \int_{\mathbb{T}^{3}}\left(\rho_{f}-\rho_{f^{\varepsilon}}\right)\left(u^{\varepsilon}-u\right)\left(u-u_{f}\right) d x d s, \\
& =: \sum_{i=1}^{4} I_{i} .
\end{aligned}
$$

- Estimate of $I_{1}$ : We first notice that $\int_{\mathbb{T}^{3}} E\left(U^{\varepsilon}\right) d x \leq \mathcal{F}\left(f^{\varepsilon}, u^{\varepsilon}\right)$, where $\mathcal{F}$ is given in (5.3). Then we obtain

$$
\begin{aligned}
I_{1}(t)= & \int_{\mathbb{T}^{3}}\left(E\left(U^{\varepsilon}\right)(t)-E\left(U_{0}\right)\right) d x+\int_{0}^{t} \int_{\mathbb{T}^{3}} \rho_{f^{\varepsilon}}\left|u^{\varepsilon}-u_{f^{\varepsilon}}\right|^{2}+\mu\left|\nabla u^{\varepsilon}\right|^{2} d x d s \\
= & \int_{\mathbb{T}^{3}} E\left(U^{\varepsilon}\right)(t) d x-\mathcal{F}\left(f^{\varepsilon}, u^{\varepsilon}\right)(t) \\
& +\mathcal{F}\left(f^{\varepsilon}, u^{\varepsilon}\right)(t)+\int_{0}^{t} \int_{\mathbb{T}^{3}} \rho_{f^{\varepsilon}}\left|u^{\varepsilon}-u_{f^{\varepsilon}}\right|^{2}+\mu\left|\nabla u^{\varepsilon}\right|^{2} d x d s-\mathcal{F}\left(f_{0}, u_{0}\right) \\
& +\mathcal{F}\left(f_{0}, u_{0}\right)-\int_{\mathbb{T}^{3}} E\left(U_{0}\right) d x \\
\leq & C\left(T^{*}\right) \varepsilon,
\end{aligned}
$$

where we used the facts that $(5.8)$ and $\mathcal{F}\left(f_{0}, u_{0}\right)=\int_{\mathbb{T}^{3}} E\left(U_{0}\right) d x$. 
- Estimate of $I_{2}$ : Straightforward computation shows that

$$
\begin{aligned}
A\left(U^{\varepsilon} \mid U\right) & =A\left(U^{\varepsilon}\right)-A(U)-d A(U)\left(U^{\varepsilon}-U\right) \\
& =\left(\begin{array}{ccc}
0 & 0 & 0 \\
\rho_{f}\left(u_{f^{\varepsilon}}-u_{f}\right) \otimes\left(u_{f}-u_{f}\right) & 0 & 0 \\
\left(u^{\varepsilon}-u\right) \otimes\left(u^{\varepsilon}-u\right) & 0 & 0
\end{array}\right) .
\end{aligned}
$$

This implies that

$$
\int_{\mathbb{T}^{3}}\left|A\left(U^{\varepsilon} \mid U\right)\right| d x=\int_{\mathbb{T}^{3}} \rho_{f^{\varepsilon}}\left|u_{f^{\varepsilon}}-u_{f}\right|^{2} d x+\int_{\mathbb{T}^{3}}\left|u^{\varepsilon}-u\right|^{2} d x \leq 2 \int_{\mathbb{T}^{3}} \mathcal{H}\left(U^{\varepsilon} \mid U\right) d x,
$$

and

$$
I_{2}(t) \leq C \int_{0}^{t} \int_{\mathbb{T}^{3}} \mathcal{H}\left(U^{\varepsilon} \mid U\right) d x d s
$$

- Estimate of $I_{3}$ : One can find that $\rho_{f^{\varepsilon}}$ and $u_{f^{\varepsilon}}$ satisfy

$$
\begin{aligned}
& \partial_{t} \rho_{f^{\varepsilon}}+\nabla \cdot\left(\rho_{f^{\varepsilon}} u_{f^{\varepsilon}}\right)=0, \\
& \partial_{t}\left(\rho_{f^{\varepsilon}} u_{f^{\varepsilon}}\right)+\nabla \cdot\left(\rho_{f^{\varepsilon}} u_{f^{\varepsilon}} \otimes u_{f^{\varepsilon}}\right)+\nabla \rho_{f^{\varepsilon}}-\rho_{f^{\varepsilon}}\left(u_{f^{\varepsilon}}-u^{\varepsilon}\right) \\
& \quad=\nabla \cdot\left(\int_{\mathbb{R}^{3}}\left(u_{f^{\varepsilon}} \otimes u_{f^{\varepsilon}}-\xi \otimes \xi+\mathbb{I}\right) f^{\varepsilon} d \xi\right),
\end{aligned}
$$

in the distribution sense on $\mathbb{T}^{3} \times\left[0, T^{*}\right)$. Then we deduce that

$$
\begin{aligned}
& \left|\int_{0}^{t} \int_{\mathbb{T}^{3}} d E(U)\left[U_{s}^{\varepsilon}+\nabla \cdot A\left(U^{\varepsilon}\right)-F\left(U^{\varepsilon}\right)\right] d x d t\right| \\
& \quad \leq\left|\int_{0}^{t} \int_{\mathbb{T}^{3}}\right| \nabla d E(U)|| \int_{\mathbb{R}^{3}}\left(u_{f^{\varepsilon}} \otimes u_{f^{\varepsilon}}-\xi \otimes \xi+\mathbb{I}\right) f^{\varepsilon} d \xi|d x d t| \\
& \quad \leq C \int_{0}^{t} \int_{\mathbb{T}^{3}}\left|\int_{\mathbb{R}^{3}}\left(u_{f^{\varepsilon}} \otimes u_{f^{\varepsilon}}-\xi \otimes \xi+\mathbb{I}\right) f^{\varepsilon} d \xi\right| d x d t .
\end{aligned}
$$

Then we now apply the same argument in [22, Lemma 4.8] to have

$$
\left|\int_{0}^{t} \int_{\mathbb{T}^{3}} d E(U)\left[U_{s}^{\varepsilon}+\nabla \cdot A\left(U^{\varepsilon}\right)-F\left(U^{\varepsilon}\right)\right] d x d s\right| \leq \sqrt{\varepsilon} C\left(T^{*}\right) .
$$

- Estimate of $I_{4}$ : The strategy is to use the third term in the dissipation. By the Cauchy-Schwartz inequality and using the fact

$$
1 \leq \min \left(\frac{1}{x}, \frac{1}{y}\right)(x+y), \quad \text { for } x, y>0,
$$

we get

$$
\begin{aligned}
& \left|\int_{\mathbb{T}^{3}}\left(\rho_{f}-\rho_{f^{\varepsilon}}\right)\left(u^{\varepsilon}-u\right)\left(u-u_{f}\right) d x\right| \\
& \quad \leq\left\|u-u_{f}\right\|_{L^{\infty}}\left(\int_{\mathbb{T}^{3}} \min \left(\frac{1}{\rho_{f}}, \frac{1}{\rho_{f^{\varepsilon}}}\right)\left|\rho_{f}-\rho_{f^{\varepsilon}}\right|^{2} d x\right)^{\frac{1}{2}}\left(\int_{\mathbb{T}^{3}}\left(\rho_{f}+\rho_{f^{\varepsilon}}\right)\left|u-u^{\varepsilon}\right|^{2} d x\right)^{\frac{1}{2}}
\end{aligned}
$$




$$
\leq C \int_{\mathbb{T}^{3}} \mathcal{H}\left(U^{\varepsilon} \mid U\right) d x+\frac{1}{4} \int_{\mathbb{T}^{3}} \rho_{f^{\varepsilon}}\left|u-u^{\varepsilon}\right|^{2} d x .
$$

On the other hand, the second term of the last inequality in (5.10) is again estimated as follows

$$
\begin{aligned}
\int_{\mathbb{T}^{3}} \rho_{f^{\varepsilon}}\left|u-u^{\varepsilon}\right|^{2} d x & =\int_{\mathbb{T}^{3}} \rho_{f^{\varepsilon}}\left|u-u_{f}+u_{f}-u_{f^{\varepsilon}}+u_{f^{\varepsilon}}-u^{\varepsilon}\right|^{2} d x \\
& \leq 2 \int_{\mathbb{T}^{3}} \rho_{f^{\varepsilon}}\left|\left(u-u_{f}\right)-\left(u^{\varepsilon}-u_{f^{\varepsilon}}\right)\right|^{2} d x+2 \int_{\mathbb{T}^{3}} \rho_{f^{\varepsilon}}\left|u_{f}-u_{f^{\varepsilon}}\right|^{2} d x,
\end{aligned}
$$

and this implies

$$
\begin{aligned}
& \frac{1}{4} \int_{\mathbb{T}^{3}} \rho_{f^{\varepsilon}}\left|u-u^{\varepsilon}\right|^{2} d x \\
& \quad \leq \frac{1}{2} \int_{\mathbb{T}^{3}} \rho_{f^{\varepsilon}}\left|u_{f}-u_{f^{\varepsilon}}\right|^{2} d x+\frac{1}{2} \int_{\mathbb{T}^{3}} \rho_{f^{\varepsilon}}\left|\left(u-u_{f}\right)-\left(u^{\varepsilon}-u_{f^{\varepsilon}}\right)\right|^{2} d x \\
& \leq \int_{\mathbb{T}^{3}} \mathcal{H}\left(U^{\varepsilon} \mid U\right) d x+\frac{1}{2} \int_{\mathbb{T}^{3}} \rho_{f^{\varepsilon}}\left|\left(u-u_{f}\right)-\left(u^{\varepsilon}-u_{f^{\varepsilon}}\right)\right|^{2} d x .
\end{aligned}
$$

This concludes that

$$
\begin{aligned}
& \left|\int_{\mathbb{T}^{3}}\left(\rho_{f}-\rho_{f^{\varepsilon}}\right)\left(u^{\varepsilon}-u\right)\left(u-u_{f}\right) d x\right| \\
& \quad \leq C \int_{\mathbb{T}^{3}} \mathcal{H}\left(U^{\varepsilon} \mid U\right) d x+\frac{1}{2} \int_{\mathbb{T}^{3}} \rho_{f^{\varepsilon}}\left|\left(u-u_{f}\right)-\left(u^{\varepsilon}-u_{f^{\varepsilon}}\right)\right|^{2} d x .
\end{aligned}
$$

From the above, we have

$$
\begin{aligned}
& \int_{\mathbb{T}^{3}} \mathcal{H}\left(U^{\varepsilon} \mid U\right)(t) d x+\mu \int_{0}^{t} \int_{\mathbb{T}^{3}}\left|\nabla\left(u-u^{\varepsilon}\right)\right|^{2} d x d s \\
& \quad+\frac{1}{2} \int_{0}^{t} \int_{\mathbb{T}^{3}} \rho_{f^{\varepsilon}}\left|\left(u_{f^{\varepsilon}}-u^{\varepsilon}\right)-\left(u_{f}-u\right)\right|^{2} d x d s \\
& \leq C \sqrt{\varepsilon}+C \int_{0}^{t} \int_{\mathbb{T}^{3}} \mathcal{H}\left(U^{\varepsilon} \mid U\right)(s) d x d s, \quad \text { for all } t \in\left[0, T^{*}\right] .
\end{aligned}
$$

We now apply Gronwall's inequality to derive that

$$
\begin{aligned}
& \int_{\mathbb{T}^{3}} \mathcal{H}\left(U^{\varepsilon} \mid U\right)(t) d x+\mu \int_{0}^{t} \int_{\mathbb{T}^{3}}\left|\nabla\left(u-u^{\varepsilon}\right)\right|^{2} d x d s \\
& \quad+\frac{1}{2} \int_{0}^{t} \int_{\mathbb{T}^{3}} \rho_{f^{\varepsilon}}\left|\left(u_{f^{\varepsilon}}-u^{\varepsilon}\right)-\left(u_{f}-u\right)\right|^{2} d x d s \leq C \sqrt{\varepsilon}
\end{aligned}
$$




\subsection{Proof of Theorem 2.2}

The entropy inequality in Proposition 5.2 and arguments in [22] yield that

$$
\begin{aligned}
& \rho_{f^{\varepsilon}} \rightarrow \rho_{f} \quad \text { in } L_{l o c}^{1}\left(0, T^{*} ; L^{1}\left(\mathbb{T}^{3}\right)\right), \\
& \rho_{f^{\varepsilon}} u_{f^{\varepsilon}} \rightarrow \rho_{f} u_{f} \quad \text { in } L_{l o c}^{1}\left(0, T^{*} ; L^{1}\left(\mathbb{T}^{3}\right)\right), \\
& \rho_{f^{\varepsilon}}\left|u_{f^{\varepsilon}}\right|^{2} \rightarrow \rho_{f}\left|u_{f}\right|^{2} \quad \text { in } L_{l o c}^{1}\left(0, T^{*} ; L^{1}\left(\mathbb{T}^{3}\right)\right), \\
& u^{\varepsilon} \rightarrow u \quad \text { in } L_{l o c}^{1}\left(0, T^{*} ; L^{2}\left(\mathbb{T}^{3}\right)\right) .
\end{aligned}
$$

Furthermore, we can also use the same argument in [22] to conclude that

$$
f^{\mathcal{E}} \rightarrow \frac{\rho_{f}}{(2 \pi)^{\frac{3}{2}}} e^{-\frac{\left|\xi-u_{f}\right|^{2}}{2}} \quad \text { in } L_{l o c}^{1}\left(0, T^{*} ; L^{1}\left(\mathbb{T}^{3} \times \mathbb{R}^{3}\right)\right) .
$$

This completes the proof.

\section{A priori estimate of asymptotic behavior (Theorem 2.3)}

In this section, we provide a long-time behavior estimate for the system (1.1)-(1.2) without diffusion, i.e., $\sigma=0$. Since the constants $\alpha$ and $\beta$ do not play any crucial role in our analysis as we mentioned before, we assume that $\alpha=\beta=1$. For the estimate of large-time behavior, we first notice that local density $\rho_{f}$ and velocity $u_{f}$ in $(1.3)$ satisfy the following hydrodynamic equations.

$$
\begin{aligned}
& \partial_{t} \rho_{f}+\nabla \cdot\left(\rho_{f} u_{f}\right)=0, \\
& \partial_{t}\left(\rho_{f} u_{f}\right)+\nabla \cdot\left(\rho_{f} u_{f} \otimes u_{f}\right)+\nabla \cdot \tilde{P}=\rho_{f}\left(u-u_{f}\right),
\end{aligned}
$$

where $\tilde{P}$ is given by

$$
\tilde{P}:=\int_{\mathbb{R}^{3}}\left(\xi-u_{f}\right) \otimes\left(\xi-u_{f}\right) f d \xi .
$$

We recall energy-fluctuation functions $\mathcal{E}_{P}, \mathcal{E}_{U}, \mathcal{E}_{F}$ and $\mathcal{E}_{I}$ :

$$
\begin{aligned}
& \mathcal{E}_{P}(t):=\frac{1}{2} \int_{\mathbb{T}^{3} \times \mathbb{R}^{3}}\left|\xi-u_{f}\right|^{2} f d x d \xi, \\
& \mathcal{E}_{U}(t):=\frac{1}{2} \int_{\mathbb{T}^{3} \times \mathbb{T}^{3}}\left|u_{f}(x)-u_{f}(y)\right|^{2} \rho_{f}(x) \rho_{f}(y) d x d y, \\
& \mathcal{E}_{F}(t):=\frac{1}{2} \int_{\mathbb{T}^{3}}\left|u-u_{c}(t)\right|^{2} d x, \\
& \mathcal{E}_{I}(t):=\frac{1}{2}\left|u_{c}(t)-\xi_{c}(t)\right|^{2} .
\end{aligned}
$$

Then we next investigate the time-evolution of the above energy-fluctuation functions.

Lemma 6.1. Let $(f, u)$ be classical solutions to the system (1.1)-(1.2) with $\sigma=0$ satisfying

$$
\lim _{|\xi| \rightarrow \infty}|\xi|^{2} f(x, \xi, t)=0, \quad(x, t) \in \mathbb{T}^{3} \times[0, T] .
$$


The following identities hold:
(i) $\frac{d \mathcal{E}_{P}}{d t}=\int_{\mathbb{T}^{3}}(\nabla \cdot \tilde{P}) \cdot u_{f} d x-4 \mathcal{E}_{P}$.
(ii) $\frac{d \mathcal{E}_{U}}{d t}=-2 \int_{\mathbb{T}^{3}}(\nabla \cdot \tilde{P}) \cdot u_{f} d x+2 \int_{\mathbb{T}^{3}} \rho_{f}\left(u-u_{f}\right) \cdot u_{f} d x$
$-2 \int_{\mathbb{T}^{3}} \rho_{f} u_{f} d x \cdot \int_{\mathbb{T}^{3}} \rho_{f}\left(u-u_{f}\right) d x$.
(iii) $\frac{d \mathcal{E}_{F}}{d t}=-\mu \int_{\mathbb{T}^{3}}|\nabla u|^{2} d x+\int_{\mathbb{T}^{3} \times \mathbb{R}^{3}}\left(u_{c}-u\right) \cdot(u-\xi) f d \xi d x$.
(iv) $\frac{d \mathcal{E}_{I}}{d t}=-2 \int_{\mathbb{T}^{3} \times \mathbb{R}^{3}}\left(u_{c}-\xi_{c}\right) \cdot(u-\xi) f d \xi d x$.

Proof. For the estimate of (i), it follows from the system (1.1) that

$$
\begin{aligned}
\frac{d \mathcal{E}_{P}}{d t} & =-\int_{\mathbb{T}^{3} \times \mathbb{R}^{3}}\left(\xi-u_{f}\right) \cdot u_{f}^{\prime} f d x d \xi+\frac{1}{2} \int_{\mathbb{T}^{3} \times \mathbb{R}^{3}}\left|\xi-u_{f}\right|^{2} \partial_{t} f d x d \xi \\
& =\frac{1}{2} \int_{\mathbb{T}^{3} \times \mathbb{R}^{3}}\left|\xi-u_{f}\right|^{2}\left(-\xi \cdot \nabla f+\nabla_{\xi} \cdot\left[\left(\xi-u_{f}\right) f\right]-\nabla_{\xi} \cdot[(u-\xi) f]\right) d x d \xi \\
& =: \sum_{i=1}^{3} \mathcal{I}_{i},
\end{aligned}
$$

where $u_{f}^{\prime}:=\frac{d}{d t} u_{f}$, and $\mathcal{I}_{i}, i=1,2,3$ are given by

$$
\begin{aligned}
\mathcal{I}_{1} & =\frac{1}{2} \int_{\mathbb{T}^{3} \times \mathbb{R}^{3}} \nabla\left(\left|\xi-u_{f}\right|^{2}\right) \cdot \xi f d x d \xi=-\int_{\mathbb{T}^{3} \times \mathbb{R}^{3}}\left(\left(\xi-u_{f}\right) \cdot \nabla u_{f}\right) \cdot \xi f d \xi d x, \\
\mathcal{I}_{2} & =-\int_{\mathbb{T}^{3} \times \mathbb{R}^{3}}\left|\xi-u_{f}\right|^{2} f d x d \xi, \\
\mathcal{I}_{3} & =\int_{\mathbb{T}^{3} \times \mathbb{R}^{3}}\left(\xi-u_{f}\right) \cdot(u-\xi) f d x d \xi=-\int_{\mathbb{T}^{3} \times \mathbb{R}^{3}} \xi \cdot\left(\xi-u_{f}\right) f d x d \xi \\
& =-\int_{\mathbb{T}^{3} \times \mathbb{R}^{3}}\left|\xi-u_{f}\right|^{2} f d x d \xi .
\end{aligned}
$$

A further integration by parts leads to

$$
\begin{aligned}
\mathcal{I}_{1} & =-\sum_{i, j=1_{\mathbb{T}^{3} \times \mathbb{R}^{3}}} \int_{i, j=1_{\mathbb{T}^{3} \times \mathbb{R}^{3}}\left(\xi^{i}-u_{f}^{i}\right) \partial_{i} u_{f}^{j} \xi^{j} f d x d \xi}\left(\xi^{i}-u_{f}^{i}\right)\left(\partial_{i} u_{f}^{j}\right)\left(\xi^{j}-u_{f}^{j}\right) f d x d \xi=\int_{\mathbb{T}^{3}}(\nabla \cdot \tilde{P}) \cdot u_{f} d x .
\end{aligned}
$$

Thus, (i) is obtained by combining (6.2) and (6.3) with the above equality. For the identity (ii), we use the hydrodynamic equations (6.1) to find 


$$
\begin{aligned}
\frac{d \mathcal{E}_{U}}{d t}= & \int_{\mathbb{T}^{3} \times \mathbb{T}^{3}}\left(u_{f}(x)-u_{f}(y)\right) \cdot\left(u_{f}^{\prime}(x)-u_{f}^{\prime}(y)\right) \rho_{f}(x) \rho_{f}(y) d x d y \\
& +\int_{\mathbb{T}^{3} \times \mathbb{T}^{3}}\left|u_{f}(x)-u_{f}(y)\right|^{2} \rho_{f}^{\prime}(x) \rho_{f}(y) d x d y \\
= & 2 \int_{\mathbb{T}^{3}} u_{f} \cdot u_{f}^{\prime} \rho_{f} d x-2\left(\int_{\mathbb{T}^{3}} \rho_{f} u_{f} d x\right) \cdot\left(\int_{\mathbb{T}^{3}} \rho_{f} u_{f}^{\prime} d x\right) \\
& +2 \int_{\mathbb{T}^{3}} \rho_{f} u_{f} \cdot\left(u_{f} \cdot \nabla u_{f}\right) d x-2\left(\int_{\mathbb{T}^{3}} \rho_{f}\left(u_{f} \cdot \nabla u_{f}\right) d x\right) \cdot\left(\int_{\mathbb{T}^{3}} \rho_{f} u_{f} d x\right) \\
= & -2 \int_{\mathbb{T}^{3}}(\nabla \cdot \tilde{P}) \cdot u_{f} d x+2 \int_{\mathbb{T}^{3}} \rho_{f}\left(u-u_{f}\right) \cdot u_{f} d x \\
& -2\left(\int_{\mathbb{T}^{3}} \rho_{f} u_{f} d x\right) \cdot\left(\int_{\mathbb{T}^{3}} \rho_{f}\left(u-u_{f}\right) d x\right),
\end{aligned}
$$

where we used the fact that $\left\|\rho_{f}\right\|_{L^{1}\left(\mathbb{T}^{3}\right)}=1$ and

$$
\rho_{f} u_{f}^{\prime}+\rho_{f} u_{f} \cdot \nabla u_{f}+\nabla \cdot \tilde{P}=\rho_{f}\left(u-u_{f}\right) .
$$

For the estimate of (iii), we use the definition of $\mathcal{E}_{F}$ and direct integration by parts to get

$$
\begin{aligned}
\frac{d \mathcal{E}_{F}}{d t}= & \int_{\mathbb{T}^{3}}\left(u-u_{c}\right) \cdot \partial_{t} u d x-u_{c}^{\prime} \cdot \int_{\mathbb{T}^{3}}\left(u-u_{c}\right) d x=\int_{\mathbb{T}^{3}}\left(u-u_{c}\right) \cdot \partial_{t} u d x \\
= & -\int_{\mathbb{T}^{3}}(u \cdot \nabla u) \cdot\left(u-u_{c}\right) d x-\int_{\mathbb{T}^{3}}\left(u-u_{c}\right) \cdot \nabla p d x \\
& +\mu \int_{\mathbb{T}^{3}}\left(u-u_{c}\right) \cdot \Delta u d x-\int_{\mathbb{T}^{3} \times \mathbb{R}^{3}}\left(u-u_{c}\right) \cdot(u-\xi) f d x d \xi \\
= & -\mu \int_{\mathbb{T}^{3}}|\nabla u|^{2} d x-\int_{\mathbb{T}^{3} \times \mathbb{R}^{3}}\left(u-u_{c}\right) \cdot(u-\xi) f d x d \xi,
\end{aligned}
$$

since $\nabla \cdot u=0$. Finally we employ the following facts

$$
\xi_{c}^{\prime}=\int_{\mathbb{T}^{3} \times \mathbb{R}^{3}}(u-\xi) f d \xi d x \quad \text { and } \quad u_{c}^{\prime}=-\int_{\mathbb{T}^{3} \times \mathbb{R}^{3}}(u-\xi) f d \xi d x,
$$

to derive the estimate of (iv)

$$
\frac{d \mathcal{E}_{I}}{d t}=\left(u_{c}-\xi_{c}\right) \cdot\left(u_{c}^{\prime}-\xi_{c}^{\prime}\right)=-2\left(u_{c}-\xi_{c}\right) \cdot \int_{\mathbb{T}^{3} \times \mathbb{R}^{3}}(u-\xi) f d \xi d x .
$$

Remark 6.1. Since $\int_{\mathbb{T}^{3}} \varrho_{f} d x \equiv 1$, we have that

$$
\int_{\mathbb{T}^{3}}\left(u_{f}-\xi_{c}\right) \rho_{f} d x=\int_{\mathbb{T}^{3} \times \mathbb{R}^{3}}\left(\xi-\xi_{c}\right) f d \xi d x=0 .
$$


As a consequence,

$$
\begin{aligned}
\mathcal{E}_{U}(t)= & \frac{1}{2} \int_{\mathbb{T}^{3} \times \mathbb{T}^{3}}\left|u_{f}(x)-u_{f}(y)\right|^{2} \rho_{f}(x) \rho_{f}(y) d x d y \\
= & \frac{1}{2} \int_{\mathbb{T}^{3} \times \mathbb{T}^{3}}\left|u_{f}(x)-\xi_{c}+\xi_{c}-u_{f}(y)\right|^{2} \rho_{f}(x) \rho_{f}(y) d x d y \\
= & \frac{1}{2} \int_{\mathbb{T}^{3} \times \mathbb{T}^{3}}\left|u_{f}(x)-\xi_{c}\right|^{2} \rho_{f}(x) \rho_{f}(y) d x d y \\
& +\frac{1}{2} \int_{\mathbb{T}^{3} \times \mathbb{T}^{3}}\left|u_{f}(y)-\xi_{c}\right|^{2} \rho_{f}(x) \rho_{f}(y) d x d y-\left(\int_{\mathbb{T}^{3}}\left(u_{f}-\xi_{c}\right) \rho_{f} d x\right)^{2} \\
= & \int_{\mathbb{T}^{3}}\left|u_{f}-\xi_{c}\right|^{2} \rho_{f} d x .
\end{aligned}
$$

Proof of Theorem 2.3. For the sake of the reader, we divide the proof into two steps.

1. In this part, we will show that

$$
\begin{aligned}
& \text { (i) } \frac{d}{d t}\left(2 \mathcal{E}_{P}+\mathcal{E}_{U}\right)=-8 \mathcal{E}_{P}-2 \mathcal{E}_{U}+2 \int_{\mathbb{T}^{3} \times \mathbb{R}^{3}}\left(\xi-\xi_{c}\right) \cdot u f d \xi d x \\
& \text { (ii) } \frac{d}{d t}\left(2 \mathcal{E}_{F}+\mathcal{E}_{I}\right)=2 \mathcal{E}_{U}+4 \mathcal{E}_{P}-2 \mu \int_{\mathbb{T}^{3}}|\nabla u|^{2} d x-2 \int_{\mathbb{T}^{3} \times \mathbb{R}^{3}}|u-\xi|^{2} f d x d \xi \\
& -2 \int_{\mathbb{T}^{3} \times \mathbb{R}^{3}}\left(\xi-\xi_{c}\right) \cdot u f d x d \xi
\end{aligned}
$$

For a detailed estimate of (i), we use Lemma 6.1 to get

$$
\begin{aligned}
\frac{d}{d t}\left(2 \mathcal{E}_{P}+\mathcal{E}_{U}\right) & =-8 \mathcal{E}_{P}+2 \int_{\mathbb{T}^{3}} \rho_{f}\left(u-u_{f}\right) \cdot\left(u_{f}-\xi_{c}\right) d x \\
& =-8 \mathcal{E}_{P}+2 \int_{\mathbb{T}^{3}} \rho_{f}\left(u-\xi_{c}\right) \cdot\left(u_{f}-\xi_{c}\right) d x-2 \int_{\mathbb{T}^{3}} \rho_{f}\left|u_{f}-\xi_{c}\right|^{2} d x \\
& =-8 \mathcal{E}_{P}-2 \mathcal{E}_{U}+2 \int_{\mathbb{T}^{3}} \rho_{f} u \cdot\left(u_{f}-\xi_{c}\right) d x
\end{aligned}
$$

where we used

$$
\int_{\mathbb{T}^{3}} \rho_{f} u_{f} d x=\xi_{c} \quad \text { and } \quad \int_{\mathbb{T}^{3}} \rho_{f}\left(u_{f}-\xi_{c}\right) d x=0 .
$$

For the second part (ii), it also follows from Lemma 6.1 that

$$
\begin{aligned}
\frac{d}{d t}\left(2 \mathcal{E}_{F}+\mathcal{E}_{I}\right)= & -2 \mu \int_{\mathbb{T}^{3}}|\nabla u|^{2} d x-2 \int_{\mathbb{T}^{3} \times \mathbb{R}^{3}}(u-\xi) \cdot\left(u-\xi_{c}\right) f d x d \xi \\
= & -2 \mu \int_{\mathbb{T}^{3}}|\nabla u|^{2} d x-2 \int_{\mathbb{T}^{3} \times \mathbb{R}^{3}}|u-\xi|^{2} f d x d \xi \\
& -2 \int_{\mathbb{T}^{3} \times \mathbb{R}^{3}} u \cdot\left(\xi-\xi_{c}\right) f d x d \xi+2 \int_{\mathbb{T}^{3} \times \mathbb{R}^{3}} \xi \cdot\left(\xi-\xi_{c}\right) f d x d \xi .
\end{aligned}
$$


On the other hand, the fourth term in the last inequality of (6.5) is estimated by

$$
2 \int_{\mathbb{T}^{3} \times \mathbb{R}^{3}} \xi \cdot\left(\xi-\xi_{c}\right) f d x d \xi=2 \int_{\mathbb{T}^{3} \times \mathbb{R}^{3}}\left|\xi-\xi_{c}\right|^{2} f d x d \xi=2 \mathcal{E}_{U}+4 \mathcal{E}_{P} .
$$

This yields the estimate of (ii).

2. We now combine two inequalities in (6.4) to find

$$
\frac{d}{d t} \mathcal{E}(t)=-4 \mathcal{E}_{P}-2 \mu \int_{\mathbb{T}^{3}}|\nabla u|^{2} d x-2 \int_{\mathbb{T}^{3} \times \mathbb{R}^{3}}|u-\xi|^{2} f d x d \xi .
$$

We set a corresponding dissipation function $\mathcal{D}(t)$ to $\mathcal{E}(t)$ :

$$
\mathcal{D}(t):=4 \mathcal{E}_{P}+2 \mu \int_{\mathbb{T}^{3}}|\nabla u|^{2} d x+2 \int_{\mathbb{T}^{3} \times \mathbb{R}^{3}}|u-\xi|^{2} f d x d \xi .
$$

Then we obtain

$$
\frac{d}{d t} \mathcal{E}(t)+\mathcal{D}(t)=0
$$

Claim. There exists a positive constant $C>0$ such that $\mathcal{E}(t) \leq C \mathcal{D}(t)$ for all $t \geq 0$.

For the proof of claim, we estimate the last term in the function $\mathcal{D}(t)$ as follows

$$
\begin{aligned}
\int_{\mathbb{T}^{3} \times \mathbb{R}^{3}}|u-\xi|^{2} f d \xi d x= & \int_{\mathbb{T}^{3} \times \mathbb{R}^{3}}\left|u-u_{c}+u_{c}-\xi_{c}+\xi_{c}-\xi\right|^{2} f d \xi d x \\
= & \int_{\mathbb{T}^{3}} \rho_{f}\left|u-u_{c}\right|^{2} d x+\left|u_{c}-\xi_{c}\right|^{2}+\int_{\mathbb{T}^{3} \times \mathbb{R}^{3}}\left|\xi_{c}-\xi\right|^{2} f d \xi d x \\
& +2 \int_{\mathbb{T}^{3} \times \mathbb{R}^{3}}\left(u-u_{c}\right) \cdot\left(u_{c}-\xi\right) f d \xi d x \\
= & 2 \mathcal{E}_{I}+2 \mathcal{E}_{P}+\mathcal{E}_{U}+\int_{\mathbb{T}^{3}} \rho_{f}\left|u-u_{c}\right|^{2} d x \\
& +2 \int_{\mathbb{T}^{3} \times \mathbb{R}^{3}}\left(u-u_{c}\right) \cdot\left(u_{c}-\xi\right) f d \xi d x
\end{aligned}
$$

where we used

$$
\int_{\mathbb{T}^{3} \times \mathbb{R}^{3}}\left(u_{c}-\xi_{c}\right) \cdot\left(\xi_{c}-\xi\right) f d \xi d x=0,
$$

and

$$
\begin{aligned}
\int_{\mathbb{T}^{3} \times \mathbb{R}^{3}}\left|\xi_{c}-\xi\right|^{2} f d \xi d x & =\int_{\mathbb{T}^{3} \times \mathbb{R}^{3}}\left|\xi_{c}-u_{f}\right|^{2} f d \xi d x+\int_{\mathbb{T}^{3} \times \mathbb{R}^{3}}\left|u_{f}-\xi\right|^{2} f d \xi d x \\
& =\mathcal{E}_{U}+2 \mathcal{E}_{P} .
\end{aligned}
$$


Furthermore we use the fact that

$$
\begin{aligned}
\int_{\mathbb{T}^{3} \times \mathbb{R}^{3}}\left|u_{c}-\xi\right|^{2} f d x d \xi & =\int_{\mathbb{T}^{3} \times \mathbb{R}^{3}}\left|u_{c}-\xi_{c}+\xi_{c}-\xi\right|^{2} f d x d \xi \\
& =\int_{\mathbb{T}^{3} \times \mathbb{R}^{3}}\left(\left|u_{c}-\xi_{c}\right|^{2}+\left|\xi_{c}-\xi\right|^{2}\right) f d x d \xi \\
& =2 \mathcal{E}_{I}+2 \mathcal{E}_{P}+\mathcal{E}_{U}
\end{aligned}
$$

to find

$$
\begin{aligned}
-4 \int_{\mathbb{T}^{3} \times \mathbb{R}^{3}}\left(u-u_{c}\right) \cdot\left(u_{c}-\xi\right) f d x d \xi & \leq 4 \int_{\mathbb{T}^{3}} \rho_{f}\left|u-u_{c}\right|^{2} d x+\int_{\mathbb{T}^{3} \times \mathbb{R}^{3}}\left|u_{c}-\xi\right|^{2} f d x d \xi \\
& =4 \int_{\mathbb{T}^{3}} \rho_{f}\left|u-u_{c}\right|^{2} d x+2 \mathcal{E}_{I}+2 \mathcal{E}_{P}+\mathcal{E}_{U} .
\end{aligned}
$$

Then it follows from (6.6) and (6.7) that

$$
2 \mathcal{E}_{I}+2 \mathcal{E}_{P}+\mathcal{E}_{U} \leq 2 \int_{\mathbb{T}^{3} \times \mathbb{R}^{3}}|u-\xi|^{2} f d x d \xi+2 \int_{\mathbb{T}^{3}} \rho_{f}\left|u-u_{c}\right|^{2} d x
$$

This deduces that

$$
\begin{aligned}
\mathcal{E}(t) & \leq \int_{\mathbb{T}^{3}}\left|u-u_{c}\right|^{2} d x+2 \int_{\mathbb{T}^{3} \times \mathbb{R}^{3}}|u-\xi|^{2} f d x d \xi+2 \int_{\mathbb{T}^{3}} \rho_{f}\left|u-u_{c}\right|^{2} d x \\
& \leq C\left(1+\left\|\rho_{f}\right\|_{L^{\infty}\left(0, \infty ; L^{3 / 2}\right)}\right) \int_{\mathbb{T}^{3}}|\nabla u|^{2} d x+2 \int_{\mathbb{T}^{3} \times \mathbb{R}^{3}}|u-\xi|^{2} f d x d \xi \\
& \leq C \mathcal{D}(t),
\end{aligned}
$$

where we used the following Sobolev inequalities

$$
\begin{aligned}
\int_{\mathbb{T}^{3}}\left|u-u_{c}\right|^{2} d x & \leq C \int_{\mathbb{T}^{3}}|\nabla u|^{2} d x, \\
\int_{\mathbb{T}^{3}} \rho_{f}\left|u-u_{c}\right|^{2} d x & \leq\left\|\rho_{f}\right\|_{L^{3 / 2}}\left\|u-u_{c}\right\|_{L^{6}}^{2} \\
& \leq C\left\|\rho_{f}\right\|_{L^{3 / 2}}\left\|u-u_{c}\right\|_{H^{1}}^{2} \leq C\left\|\rho_{f}\right\|_{L^{\infty}\left(0, \infty ; L^{3 / 2}\right)}\|\nabla u\|_{L^{2}}^{2} .
\end{aligned}
$$

This yields the proof of claim, and we have

$$
\frac{d}{d t} \mathcal{E}(t)+C \mathcal{E}(t) \leq 0, \quad t \geq 0,
$$

for some positive constant $C>0$. This completes the proof.

\section{Conflict of interest statement}

The authors do not have any conflict of interest. 


\section{Acknowledgements}

JAC was partially supported by the project MTM2011-27739-C04-02 DGI (Spain) and 2009-SGR-345 from AGAUR-Generalitat de Catalunya. JAC acknowledges support from the Royal Society by a Wolfson Research Merit Award. YPC was supported by Basic Science Research Program through the National Research Foundation of Korea funded by the Ministry of Education, Science and Technology (Ref. 2012R1A6A3A03039496). JAC and YPC were supported by Engineering and Physical Sciences Research Council grants with references EP/K008404/1 (individual grant) and EP/I019111/1 (platform grant). The work of TK was supported by the Norwegian Research Council (proj. 205738).

\section{Appendix A. Proof of Lemma 5.1}

In this part, we provide the proof of Lemma 5.1. It follows from (5.9) that

$$
\begin{aligned}
\frac{d}{d t} \int_{\mathbb{T}^{3}} \mathcal{H}(V \mid U) d x= & \int_{\mathbb{T}^{3}} \partial_{t} E(V) d x-\int_{\mathbb{T}^{3}} d E(U)\left(V_{t}+\nabla \cdot A(V)-F(V)\right) d x \\
& +\int_{\mathbb{T}^{3}} d^{2} E(U) \nabla \cdot A(U)(V-U)+d E(U) \nabla \cdot A(V) d x \\
& -\int_{\mathbb{T}^{3}} d^{2} E(U) F(U)(V-U)+d E(U) F(V) d x \\
= & : \sum_{i=1}^{4} I_{i} .
\end{aligned}
$$

Using integration by parts, we find

$$
\begin{aligned}
I_{3} & =\int_{\mathbb{T}^{3}}(\nabla d E(U)):(d A(U)(V-U)-A(V)) d x \\
& =-\int_{\mathbb{T}^{3}}(\nabla d E(U)):(A(V \mid U)+A(U)) d x \\
& =-\int_{\mathbb{T}^{3}}(\nabla d E(U)): A(V \mid U) d x .
\end{aligned}
$$

Here we used the fact that

$$
\int_{\mathbb{T}^{3}}(\nabla d E(U)): A(U) d x=\int_{\mathbb{T}^{3}} \nabla \cdot Q(U) d x=0,
$$

where $Q$ is an entropy flux function given by

$$
Q_{i}(U):=\sum_{k} A_{k i}(U) d_{k} E(U)
$$

For the estimate $I_{4}$, we claim that the following identity holds.

$$
\begin{gathered}
\int_{\mathbb{T}^{3}} d^{2} E(U) F(U)(V-U)+d E(U) F(V) d x \\
=-\int_{\mathbb{T}^{3}} \varrho_{\bar{f}}\left|u_{\bar{f}}-\bar{u}\right|^{2} d x-\mu \int_{\mathbb{T}^{3}}|\nabla \bar{u}|^{2} d x
\end{gathered}
$$




$$
\begin{aligned}
& +\int_{\mathbb{T}^{3}} \varrho_{\bar{f}}\left|\left(u_{f}-u\right)-\left(u_{\bar{f}}-\bar{u}\right)\right|^{2} d x+\mu \int_{\mathbb{T}^{3}}|\nabla(u-\bar{u})|^{2} d x \\
& +\int_{\mathbb{T}^{3}}\left(\varrho_{f}-\varrho_{\bar{f}}\right)(\bar{u}-u)\left(u_{f}-u\right) d x .
\end{aligned}
$$

Proof of Claim. We first notice that

$$
d E(U)=\left(\begin{array}{c}
\log \varrho_{f}+1-\frac{m_{f}^{2}}{2 \varrho_{f}^{2}} \\
\frac{m_{f}}{\varrho_{f}}
\end{array}\right) \quad \text { and } \quad d^{2} E(U)=\left(\begin{array}{ccc}
* & -\frac{m_{f}}{\varrho_{f}^{2}} & 0 \\
* & \frac{1}{\varrho_{f}} & 0 \\
0 & 0 & 1
\end{array}\right) .
$$

Then by direct calculation, we have

$$
\begin{aligned}
& \int_{\mathbb{T}^{3}} d^{2} E(U) F(U)(V-U) d x \\
& =\int_{\mathbb{T}^{3}} \varrho_{f}\left[u_{f} \bar{u}-u_{f} u-u \bar{u}+u^{2}\right]+\varrho_{\bar{f}}\left[-u_{f} u+u_{f}^{2}+u_{\bar{f}} u-u_{\bar{f}} u_{f}\right] d x \\
& \quad+\int_{\mathbb{T}^{3}}(\bar{u}-u) \mu \Delta u-(\bar{u}-u) \nabla p d x
\end{aligned}
$$

and moreover

$$
\begin{aligned}
\int_{\mathbb{T}^{3}} d E(U) F(V) d x= & \int_{\mathbb{T}^{3}} \varrho_{\bar{f}}\left[u_{f} \bar{u}-u_{\bar{f}} u_{f}+u_{\bar{f}} u-\bar{u} u\right] d x \\
& +\int_{\mathbb{T}^{3}} \mu u \Delta \bar{u}-u \nabla \bar{p} d x .
\end{aligned}
$$

By combining (A.2)-(A.3), and using that $\nabla \cdot u=\nabla \cdot \bar{u}=0$, we obtain

$$
\begin{aligned}
& \int_{\mathbb{T}^{3}} d^{2} E(U) F(U)(V-U)+d E(U) F(V) d x \\
& =\int_{\mathbb{T}^{3}} \varrho_{\bar{f}}\left[-u_{f} u+u_{f}^{2}-2 u_{\bar{f}} u_{f}+2 u_{\bar{f}} u+u_{f} \bar{u}-\bar{u} u\right] d x \\
& \quad+\int_{\mathbb{T}^{3}} \varrho_{f}\left[u_{f} \bar{u}-u_{f} u-u \bar{u}+u^{2}\right] d x \\
& \quad+\int_{\mathbb{T}^{3}} \mu[u \Delta \bar{u}+\bar{u} \Delta u-u \Delta u] d x=: J_{1}+J_{2}+J_{3} .
\end{aligned}
$$

By adding and subtracting, we rewrite $J_{1}$ as follows

$$
\begin{aligned}
J_{1} & =\int_{\mathbb{T}^{3}} \varrho_{\bar{f}}\left[-u_{f} u+u_{f}^{2}-2 u_{\bar{f}} u_{f}+2 u_{\bar{f}} u+u_{f} \bar{u}-\bar{u} u\right] d x \\
& =\int_{\mathbb{T}^{3}} \varrho_{\bar{f}}\left[-2\left(u_{\bar{f}}-\bar{u}\right)\left(u_{f}-u\right)-\bar{u}\left(u_{f}-u\right)-u_{f} u+u_{f}^{2}\right] d x .
\end{aligned}
$$


Next, we add and subtract $\varrho_{\bar{f}}\left|u_{f}-u\right|^{2}$ to discover

$$
\begin{aligned}
J_{1}= & \int_{\mathbb{T}^{3}} \varrho_{\bar{f}}\left[-2\left(u_{\bar{f}}-\bar{u}\right)\left(u_{f}-u\right)+\left|u_{f}-u\right|^{2}\right] d x \\
& +\int_{\mathbb{T}^{3}} \varrho_{\bar{f}}\left[-\left|u_{f}-u\right|^{2}-\bar{u}\left(u_{f}-u\right)-u_{f} u+u_{f}^{2}\right] d x \\
= & \int_{\mathbb{T}^{3}} \varrho_{\bar{f}}\left|\left(u_{\bar{f}}-\bar{u}\right)-\left(u_{f}-u\right)\right|^{2} d x-\int_{\mathbb{T}^{3}} \varrho_{\bar{f}}\left|u_{\bar{f}}-\bar{u}\right|^{2} d x \\
& +\int_{\mathbb{T}^{3}} \varrho_{\bar{f}}\left[u_{f} u-u^{2}-\bar{u} u_{f}+\bar{u} u\right] d x .
\end{aligned}
$$

As a consequence, we find that

$$
\begin{aligned}
J_{1}+J_{2}= & \int_{\mathbb{T}^{3}} \varrho_{\bar{f}}\left|\left(u_{\bar{f}}-\bar{u}\right)-\left(u_{f}-u\right)\right|^{2} d x-\int_{\mathbb{T}^{3}} \varrho_{\bar{f}}\left|u_{\bar{f}}-\bar{u}\right|^{2} d x \\
& +\int_{\mathbb{T}^{3}}\left(\varrho_{\bar{f}}-\varrho_{f}\right)\left[u_{f} u-u^{2}-\bar{u} u_{f}+\bar{u} u\right] d x \\
= & \int_{\mathbb{T}^{3}} \varrho_{\bar{f}}\left|\left(u_{\bar{f}}-\bar{u}\right)-\left(u_{f}-u\right)\right|^{2} d x-\int_{\mathbb{T}^{3}} \varrho_{\bar{f}}\left|u_{\bar{f}}-\bar{u}\right|^{2} d x \\
& +\int_{\mathbb{T}^{3}}\left(\varrho_{\bar{f}}-\varrho_{f}\right)(u-\bar{u})\left(u_{f}-u\right) d x .
\end{aligned}
$$

Next, we apply integration by parts to write $J_{3}$ in the form

$$
J_{3}=\int_{\mathbb{T}^{3}} \mu[u \Delta \bar{u}+\bar{u} \Delta u-u \Delta u] d x=-\mu \int_{\mathbb{T}^{3}}|\nabla \bar{u}|^{2} d x+\mu \int_{\mathbb{T}^{3}}|\nabla(u-\bar{u})|^{2} d x .
$$

By setting (A.5) and (A.6) in (A.4), we obtain (A.1).

\section{References}

[1] H.-O. Bae, Y.-P. Choi, S.-Y. Ha, M.-J. Kang, Global existence of strong solution for the Cucker-Smale-Navier-Stokes system, J. Differ. Equ. 257 (2014) 2225-2255.

[2] H.-O. Bae, Y.-P. Choi, S.-Y. Ha, M.-J. Kang, Asymptotic flocking dynamics of Cucker-Smale particles immersed in compressible fluids, Discrete Contin. Dyn. Syst., Ser. A 34 (2014) 4419-4458.

[3] L. Boudin, L. Desvillettes, C. Grandmont, A. Moussa, Global existence of solution for the coupled Vlasov and Naiver-Stokes equations, Differ. Integral Equ. 22 (2009) 1247-1271.

[4] J.A. Cañizo, J.A. Carrillo, J. Rosado, A well-posedness theory in measures for some kinetic models of collective motion, Math. Models Methods Appl. Sci. 21 (2011) 515-539.

[5] J.A. Carrillo, R. Duan, A. Moussa, Global classical solutions close to the equilibrium to the Vlasov-Fokker-Planck-Euler system, Kinet. Relat. Models 4 (2011) 227-258.

[6] J.A. Carrillo, M. Fornasier, J. Rosado, G. Toscani, Asymptotic flocking dynamics for the kinetic Cucker-Smale model, SIAM J. Math. Anal. 42 (2010) 218-236.

[7] J.A. Carrillo, M. Fornasier, G. Toscani, F. Vecil, Particle, kinetic, and hydrodynamic models of swarming, in: G. Naldi, L. Pareschi, G. Toscani (Eds.), Mathematical Modeling of Collective Behavior in Socio-Economic and Life Sciences, in: Modelling and Simulation in Science and Technology, Birkhäuser, 2010, pp. 297-336.

[8] J.A. Carrillo, T. Goudon, Stability and asymptotic analysis of a fluid-particle interaction model, Commun. Partial Differ. Equ. 31 (2006) 1349-1379.

[9] F. Cucker, S. Smale, Emergent behavior in flocks, IEEE Trans. Autom. Control 52 (2007) 852-862.

[10] C.M. Dafermos, The second law of thermodynamics and stability, Arch. Ration. Mech. Anal. 70 (1979) 167-179. 
[11] P. Degond, Global existence of smooth solutions for the Vlasov-Fokker-Planck equation in 1 and 2 space dimensions, Ann. Sci. Éc. Norm. Super. 19 (1986) 519-542.

[12] V. Girault, P.-A. Raviart, Finite Element Methods for Navier-Stokes Equations. Theory and Algorithms, Springer Series in Computational Mathematics, vol. 5, Springer-Verlag, Berlin, 1986.

[13] R. Glassey, The Cauchy Problem in Kinetic Theory, Society for Industrial and Applied Mathematics, Philadelphia, 1996.

[14] P. Goncalves, C. Landim, C. Toninelli, Hydrodynamic limit for a particle system with degenerate rates, Ann. Inst. Henri Poincaré Probab. Stat. 45 (4) (2009) 887-909.

[15] T. Goudon, L. He, A. Moussa, P. Zhang, The Navier-Stokes-Vlasov-Fokker-Planck system near equilibrium, SIAM J. Math. Anal. 42 (2010) $2177-2202$.

[16] T. Goudon, P.-E. Jabin, A. Vasseur, Hydrodynamic limit for the Vlasov-Navier-Stokes equations: I. Light particles regime, Indiana Univ. Math. J. 53 (2004) 1495-1515.

[17] T. Goudon, P.-E. Jabin, A. Vasseur, Hydrodynamic limit for the Vlasov-Navier-Stokes equations: II. Fine particles regime, Indiana Univ. Math. J. 53 (2004) 1517-1536.

[18] K. Hamdache, Global existence and large time behavior of solutions for the Vlasov-Stokes equations, Jpn. J. Ind. Appl. Math. 15 (1998) $51-74$.

[19] S.-Y. Ha, J.-G. Liu, A simple proof of Cucker-Smale flocking dynamics and mean field limit, Commun. Math. Sci. 7 (2009) $297-325$.

[20] S.-Y. Ha, E. Tadmor, From particle to kinetic and hydrodynamic description of flocking, Kinet. Relat. Models 1 (2008) 415-435.

[21] T.K. Karper, A. Mellet, K. Trivisa, Existence of weak solutions to kinetic flocking models, SIAM J. Math. Anal. 45 (1) (2013) $215-243$.

[22] T.K. Karper, A. Mellet, K. Trivisa, Hydrodynamic limit of the kinetic Cucker-Smale flocking model, Math. Models Methods Appl. Sci. (2014), http://dx.doi.org/10.1142/S0218202515500050.

[23] T.K. Karper, A. Mellet, K. Trivisa, On strong local alignment in the kinetic Cucker-Smale model, in: Hyperbolic Conservation Laws and Related Analysis with Applications, in: Springer Proc. Math. Stat., vol. 49, 2014, pp. 227-242.

[24] C. Landim, Hydrodynamic limit of interacting particle systems, in: School and Conference on Probability Theory, in: ICTP Lect. Notes, vol. XVII, Abdus Salam Int. Cent. Theoret. Phys., Trieste, 2004, 57100 (electronic).

[25] A. Majda, Compressible Fluid Flow and Systems of Conservation Laws in Several Space Variables, Applied Mathematical Sciences, vol. 53, Springer-Verlag, New York, 1984.

[26] A. Mellet, A. Vasseur, Global weak solutions for a Vlasov-Fokker-Planck/Navier-Stokes system of equations, Math. Models Methods Appl. Sci. 17 (2007) 1039-1063.

[27] A. Mellet, A. Vasseur, Asymptotic analysis for a Vlasov-Fokker-Planck/compressible Navier-Stokes equations, Commun. Math. Phys. 281 (2008) 573-596.

[28] S. Motsch, E. Tadmor, A new model for self-organized dynamics and its flocking behavior, J. Stat. Phys. 141 (5) (2011) $923-947$.

[29] H.T. Yau, Relative entropy and hydrodynamics of Ginzburg-Landau models, Lett. Math. Phys. 22 (1) (1991) 6380. 